\title{
SU(2) and SU(1,1) Algebra Eigenstates: A Unified Analytic Approach to Coherent and Intelligent States
}

\author{
Constantin Brif * \\ Department of Physics, Technion - Israel Institute of Technology, Haifa 32000, Israel
}

\begin{abstract}
We introduce the concept of algebra eigenstates which are defined for an arbitrary Lie group as eigenstates of elements of the corresponding complex Lie algebra. We show that this concept unifies different definitions of coherent states associated with a dynamical symmetry group. On the one hand, algebra eigenstates include different sets of Perelomov's generalized coherent states. On the other hand, intelligent states (which are squeezed states for a system of general symmetry) also form a subset of algebra eigenstates. We develop the general formalism and apply it to the $\mathrm{SU}(2)$ and $\mathrm{SU}(1,1)$ simple Lie groups. Complete solutions to the general eigenvalue problem are found in the both cases, by a method that employs analytic representations of the algebra eigenstates. This analytic method also enables us to obtain exact closed expressions for quantum statistical properties of an arbitrary algebra eigenstate. Important special cases such as standard coherent states and intelligent states are examined and relations between them are studied by using their analytic representations.
\end{abstract}

\section{INTRODUCTION}

Coherent states (CS) associated with various Lie groups have been successfully used in the last decades in many problems of quantum physics (Klauder and Skagerstam, 1985; Perelomov, 1986; Zhang et al., 1990). There are three different group-theoretic approaches to CS (Zhang et al., 1990). These approaches follow three possible definitions of the familiar Glauber CS $|\alpha\rangle$ of a harmonic oscillator (Glauber, 1963). Perelomov (1972, 1977), Gilmore (1972, 1974) and Rasetti (1975) have developed the formalism in which CS are generated by the action of group elements on a reference state of a group representation Hilbert space. In the second approach one deals with eigenstates of a lowering group generator (Barut and Girardello, 1971). The third definition of CS is related to the optimization of uncertainty relations for Hermitian generators of a group (Schrödinger, 1926). States that minimize uncertainty relations are called intelligent states (IS) (Aragone et al., 1974, 1976; Ruschin and Ben-Aryeh, 1976; Vanden-Bergh and DeMeyer, 1978) or minimum-uncertainty states. Different definitions coincide only in the special case of the Heisenberg-Weyl group (Weyl, 1950) that is the dynamical symmetry group of a quantized harmonic oscillator; then one obtains the Glauber CS $|\alpha\rangle$ (Glauber, 1963). For more complicated groups, e.g., for $\mathrm{SU}(2)$ and $\mathrm{SU}(1,1)$, the different approaches lead to distinct states.

Relations between various sets of coherent and intelligent states have been studied in a number of recent works (Wodkiewicz and Eberly, 1985; Trifonov, 1994; Brif and Ben-Aryeh, 1994a). In the present paper we continue and extend this study by developing a group-theoretic formalism that provides a unified description of different types of coherent and intelligent states. We introduce the concept of algebra eigenstates (AES) which are defined for an arbitrary Lie group as eigenstates

\footnotetext{
${ }^{*}$ E-mail: costya@physics.technion.ac.il. URL address: http://www.technion.ac.il/ ${ }^{\text {briff. }}$
} 
of elements of the corresponding complex Lie algebra. This general approach incorporates in a simple way the three different definitions mentioned above. Different sets of Perelomov's CS associated with a Lie group can be equivalently defined as the AES for this group. The IS for Hermitian generators of a group also form a subset of the AES. The algebra-eigenstate formalism enables us to use powerful analytic methods for treating different types of states in a unified way. Recently, we have also considered (Brif, 1996) the two-photon AES which provide a unified analytic approach to single-mode squeezing. Similar ideas have been also discussed recently by Puri and Agarwal (1996) and by Trifonov (1996a,b).

In this work we apply the general formalism to the $\mathrm{SU}(2)$ and $\mathrm{SU}(1,1)$ simple Lie groups. We use analytic representations based on the standard sets of Perelomov's CS for these groups. In the $\mathrm{SU}(1,1)$ case an alternative analytic representation based on the Barut-Girardello states (Barut and Girardello, 1971) is also used. The eigenvalue equation that determines the AES is converted by means of an analytic representation into a linear homogeneous differential equation. Solving this equation, we obtain the complete solution of the general eigenvalue problem. Then the theory of analytic functions is applied for studying quantum statistical properties of the AES and relations between their subsets.

\section{THE GENERAL THEORY OF THE ALGEBRA EIGENSTATES}

\subsection{Definitions}

Let $G$ be an arbitrary Lie group and $T$ its unitary irreducible representation acting on the Hilbert space $\mathcal{H}$. Let $Y$ be the complex Lie algebra of the group $G$ (in what follows we will call algebra the complex extension of the real algebra, i.e., the set of all linear combinations of elements of the real algebra with complex coefficients). If we choose a basis $\left\{y_{1}, y_{2}, \ldots, y_{p}\right\}$ for the $p$-dimensional Lie algebra $Y$, then an element of the complex algebra can be written as the Euclidean scalar product in the $p$-dimensional vector space,

$$
(\vec{\beta} \cdot \vec{y})=\beta_{1} y_{1}+\beta_{2} y_{2}+\cdots+\beta_{p} y_{p},
$$

where $\beta_{1}, \beta_{2}, \ldots, \beta_{p}$ are arbitrary complex coefficients. Then, the AES are defined by the eigenvalue equation:

$$
(\vec{\beta} \cdot \vec{y})|\Psi(\lambda, \vec{\beta})\rangle=\lambda|\Psi(\lambda, \vec{\beta})\rangle, \quad|\Psi(\lambda, \vec{\beta})\rangle \in \mathcal{H} .
$$

Admissible values of $\vec{\beta}$ and $\lambda$ depend on the structure of the group and will be determined for all particular situations that will be considered in the text. A special case of the eigenvalue equation (2.2) is the time-independent Schrödinger equation for Hamiltonians which are linear combinations of group generators. However, we will see that apart from this special case the eigenvalue equation (2.2) contains other important cases and has a fundamental meaning in the theory of coherent and intelligent states.

\subsection{Generalized coherent states}

Now we turn back to the Perelomov definition of the generalized CS. By choosing a fixed normalized reference state $\left|\Psi_{0}\right\rangle \in \mathcal{H}$, one can define the system of states $\left\{\left|\Psi_{g}\right\rangle\right\}$,

$$
\left|\Psi_{g}\right\rangle=T(g)\left|\Psi_{0}\right\rangle, \quad g \in G
$$

which is called the coherent-state system. The isotropy (or maximum-stability) subgroup $H \subset G$ consists of all the group elements $h$ that leave the reference state invariant up to a phase factor,

$$
T(h)\left|\Psi_{0}\right\rangle=e^{i \phi(h)}\left|\Psi_{0}\right\rangle, \quad\left|e^{i \phi(h)}\right|=1, \quad h \in H .
$$


For every element $g \in G$, there is a unique decomposition of $g$ into a product of two group elements, one in $H$ and the other in the quotient (or coset) space $G / H$,

$$
g=\Omega h, \quad g \in G, \quad h \in H, \quad \Omega \in G / H .
$$

It is clear that group elements $g$ and $g^{\prime}$ with different $h$ and $h^{\prime}$ but with the same $\Omega$ produce the coherent states which differ only by a phase factor: $\left|\Psi_{g}\right\rangle=e^{i \delta}\left|\Psi_{g^{\prime}}\right\rangle$, where $\delta=\phi(h)-\phi\left(h^{\prime}\right)$. Therefore a coherent state $\left|\Psi_{\Omega}\right\rangle$ is determined by a point $\Omega=\Omega(g)$ in the quotient space $G / H$.

One can see from this group-theoretic procedure for the generation of the CS, that the choice of the reference state $\left|\Psi_{0}\right\rangle$ firmly determines the structure of the coherent-state set. Different choices of the reference state give different sets of the CS. An important class of coherent-state sets corresponds to the quotient spaces $G / H$ which are homogeneous Kählerian manifolds. Then $G / H$ can be considered as the phase space of a classical dynamical system, and the mapping $\Omega \rightarrow\left|\Psi_{\Omega}\right\rangle\left\langle\Psi_{\Omega}\right|$ is the quantization for this system (Berezin, 1975). The usually used sets of the CS (the standard sets, as we refer to them) correspond to the cases when an extreme state of the representation Hilbert space (e.g., the vacuum state of an oscillator or the lowest spin state) is chosen as the reference state (Zhang et al., 1990). In general, this choice of the reference state leads to the sets consisting of states with properties closest to those of classical states (Perelomov, 1986).

The isotropy subalgebra $X$ is defined as the set of elements $\{x\}, x \in Y$, such that

$$
x\left|\Psi_{0}\right\rangle=\lambda\left|\Psi_{0}\right\rangle
$$

where $\lambda$ is a complex eigenvalue. By acting with $T(g)$ on both sides of equation (2.6), we obtain

$$
T(g) x T^{-1}(g) T(g)\left|\Psi_{0}\right\rangle=\lambda T(g)\left|\Psi_{0}\right\rangle .
$$

This leads to the eigenvalue equation

$$
y\left|\Psi_{g}\right\rangle=\lambda\left|\Psi_{g}\right\rangle
$$

where $\left|\Psi_{g}\right\rangle=T(g)\left|\Psi_{0}\right\rangle$ is a coherent state, and $y=T(g) x T^{-1}(g)$ is an element of the algebra $Y$. We see that, for nontrivial $X$, Perelomov's generalized CS $\left|\Psi_{g}\right\rangle$ can be defined as the AES, and a specific set of the CS is obtained for the appropriate choice of the parameters $\beta$ 's. More precisely, let a state $|\Psi(\lambda, \vec{\beta})\rangle$ belong to a specific set of the CS corresponding to the reference state $\left|\Psi_{0}\right\rangle$ that satisfies equation (2.6). Then the parameters $\beta$ 's must satisfy the condition $(\vec{\beta} \cdot \vec{y})=T(g) x T^{-1}(g), \forall g \in G$. Note that the definition of the AES does not depend explicitly on the choice of the reference state $\left|\Psi_{0}\right\rangle$. Hence it is possible to treat the CS defined as the AES in a quite general way, regardless of the set to which they belong.

\subsection{Analytic representations}

An important property of the generalized CS is the identity resolution:

$$
\int d \mu(\Omega)\left|\Psi_{\Omega}\right\rangle\left\langle\Psi_{\Omega}\right|=I
$$

where $I$ is the identity operator in the Hilbert space $\mathcal{H}$, and $d \mu(\Omega)$ is the invariant measure in the homogeneous quotient space $G / H$. Then any state $|\Psi\rangle \in \mathcal{H}$ can be expanded in the coherent-state basis $\left|\Psi_{\Omega}\right\rangle$,

$$
|\Psi\rangle=\int d \mu(\Omega) f(\Omega)\left|\Psi_{\Omega}\right\rangle
$$

where $f(\Omega)=\left\langle\Psi_{\Omega} \mid \Psi\right\rangle$, and

$$
\langle\Psi \mid \Psi\rangle=\int d \mu(\Omega)|f(\Omega)|^{2}<\infty
$$


Now, let us represent all the AES in the standard coherent-state basis. In what follows we will consider only the simplest cases in which the quotient space $G / H$ corresponding to the standard set is a homogeneous Kählerian manifold that can be parametrized by a single complex number $\zeta$, so we write the standard generalized CS $\left|\Psi_{\Omega}\right\rangle$ in the form $|\zeta\rangle$. Then equation (2.10) reads for the AES:

$$
|\Psi(\lambda, \vec{\beta})\rangle=\int d \mu(\zeta) f\left(\lambda, \vec{\beta} ; \zeta^{*}\right)|\zeta\rangle
$$

The function $f(\lambda, \vec{\beta} ; \zeta)=\left\langle\zeta^{*} \mid \Psi(\lambda, \vec{\beta})\right\rangle$ can be factorized as $f(\lambda, \vec{\beta} ; \zeta)=\mathcal{R}(\zeta) \Lambda(\lambda, \vec{\beta} ; \zeta)$. Here $\mathcal{R}(\zeta)$ is a normalization factor such that $\Lambda(\lambda, \vec{\beta} ; \zeta)$ is an analytic function of $\zeta$ defined on the whole complex plane or on part of it. Such analytic representations are well studied (Fock, 1928; Bargmann, 1961; Segal, 1962) for the standard coherent-state bases of the simplest Lie groups (Perelomov, 1986). In these simplest cases the elements of the Lie algebra act in the Hilbert space of analytic functions as linear differential operators. Then the eigenvalue equation (2.2) is converted into a linear homogeneous differential equation. Solving it, we obtain the analytic functions $\Lambda(\lambda, \vec{\beta} ; \zeta)$ representing the $\operatorname{AES}|\Psi(\lambda, \vec{\beta})\rangle$ in the standard coherent-state basis $|\zeta\rangle$. The requirement of the analyticity provides us with the domain of admissible values of $\lambda$ and $\vec{\beta}$. The knowledge of the function $\Lambda(\lambda, \vec{\beta} ; \zeta)$ enables us to calculate properties of the corresponding state. In this paper we will use these analytic representations for finding the expansion of the AES in the orthonormal basis of the representation Hilbert space, including the explicit calculation of the normalization factor. We also will demonstrate how this analytic method can be used for obtaining exact analytic expressions for some expectation values over the AES.

\subsection{Intelligent states}

The standard set of Perelomov's CS is a particular case of the wide system of the AES. Other particular cases of the AES are the sets of the ordinary and generalized IS. Any two quantum observables (Hermitian operators in the Hilbert space) $A$ and $B$ obey the Schrödinger-Robertson uncertainty relation

$$
(\Delta A)^{2}(\Delta B)^{2} \geq \frac{1}{4}\left(|\langle[A, B]\rangle|^{2}+4 \sigma_{A B}^{2}\right),
$$

where the variance of $A$ is $(\Delta A)^{2}=\left\langle A^{2}\right\rangle-\langle A\rangle^{2},(\Delta B)^{2}$ is defined similarly, the covariance of $A$ and $B$ is $\sigma_{A B}=\frac{1}{2}\langle A B+B A\rangle-\langle A\rangle\langle B\rangle$, and the expectation values are taken over an arbitrary state in the Hilbert space. When the covariance of $A$ and $B$ vanishes, $\sigma_{A B}=0$, the Schrödinger-Robertson uncertainty relation is reduced to the Heisenberg uncertainty relation,

$$
(\Delta A)^{2}(\Delta B)^{2} \geq \frac{1}{4}|\langle[A, B]\rangle|^{2} .
$$

The ordinary IS (Aragone et al., 1974, 1976) provide an equality in the Heisenberg uncertainty relation (2.14), while the generalized IS (Trifonov, 1994) do so in the Schrödinger-Robertson uncertainty relation (2.13). It is clear that the ordinary IS form a subset of the generalized IS. The generalized IS for operators $A$ and $B$ are determined from the eigenvalue equation (Trifonov, 1994; Puri, 1994)

$$
(\eta A+i B)|\lambda, \eta\rangle=\lambda|\lambda, \eta\rangle
$$

where the parameter $\eta$ is an arbitrary complex number, and $\lambda$ is a complex eigenvalue. For the particular case of real $\eta$, the eigenvalue equation (2.15) determines the ordinary IS for operators $A$ and $B$. Then the equation can be written in the form (Jackiw, 1968)

$$
(A+i \gamma B)|\lambda, \gamma\rangle=\lambda|\lambda, \gamma\rangle
$$

where $\gamma$ is a real parameter. By comparing equations (2.15) and (2.16) with equation (2.2), we see that the IS for any two Hermitian group generators form a subset of the AES of the group. 
Eigenstates of a lowering group generator (Barut and Girardello, 1971; Dodonov et al., 1974; Hillery, 1987, 1989; Agarwal, 1988; Bužek, 1990; Brif and Ben-Aryeh, 1994b; Brif, 1995), being a special case of the IS, are a simple example of the AES.

The generalized IS for position and momentum of a harmonic oscillator coincide (Trifonov, 1994) with the canonical squeezed states (Stoler, 1970, 1971; Yuen, 1976), and they also are referred to as the correlated coherent states (Dodonov et al., 1980). The concept of squeezing is naturally related also to the IS associated with more complicated Lie groups (Wodkiewicz and Eberly, 1985; Hillery, 1987, 1989; Nieto and Truax, 1993; Trifonov, 1994). At the last years there is a great interest in the IS (Wodkiewicz and Eberly, 1985; Agarwal and Puri, 1990; Bergou et al., 1991; Hillery and Mlodinow, 1993; Nieto and Truax, 1993; Trifonov, 1994; Brif and Ben-Aryeh, 1994a; Yu and Hillery, 1994; Prakash and Agarwal, 1994, 1995; Gerry and Grobe, 1995; Puri and Agarwal, 1996; Luis and Peřina, 1996; Brif and Ben-Aryeh, 1996; Brif and Mann 1996a,b), especially for generators of the $\mathrm{SU}(2)$ and $\mathrm{SU}(1,1)$ Lie groups. The $\mathrm{SU}(2)$ and $\mathrm{SU}(1,1)$ IS have been recently shown to be very useful for improving the accuracy of interferometric measurements (Hillery and Mlodinow, 1993; Brif and Ben-Aryeh, 1996; Brif and Mann 1996a,b).

The investigation of the AES yields the most full information on the IS for generators of the corresponding Lie group. This information is of great importance in quantum optical applications of the IS. The most convenient way for examining different subsets of the AES and the relations between them is the construction of the analytic representation of the AES in the standard coherent-state basis. Actually, the idea to use such an analytic representation has been recently applied (Trifonov, 1994; Brif and Ben-Aryeh, 1994a) to the SU(1,1) IS. As has been recently shown by Brif and Mann (1996a,b), the use of these representations is a powerful method for obtaining closed analytic expressions for various properties of the IS. In the present work the analytic representations are obtained for arbitrary AES of the $\mathrm{SU}(2)$ and $\mathrm{SU}(1,1)$ Lie groups. These representations are used for finding the expansion of the AES in the corresponding orthonormal basis, including the calculation of exact analytic expressions for the normalization factor and for some quantum expectation values.

\section{THE SU(2) ALGEBRA EIGENSTATES}

In this section we discuss the AES for the $\mathrm{SU}(2)$ group which is the most elementary compact non-Abelian simple Lie group. The corresponding Lie algebra is spanned by the three operators $\left\{J_{1}, J_{2}, J_{3}\right\}$,

$$
\left[J_{1}, J_{2}\right]=i J_{3}, \quad\left[J_{2}, J_{3}\right]=i J_{1}, \quad\left[J_{3}, J_{1}\right]=i J_{2} .
$$

It is convenient to use raising and lowering operators $J_{ \pm}=J_{1} \pm i J_{2}$ which satisfy the following commutation relations

$$
\left[J_{3}, J_{ \pm}\right]= \pm J_{ \pm}, \quad\left[J_{-}, J_{+}\right]=-2 J_{3}
$$

The Casimir operator $J^{2}=J_{1}^{2}+J_{2}^{2}+J_{3}^{2}$ for any unitary irreducible representation is the identity operator times a number: $J^{2}=j(j+1) I$. Thus a representation of the $\mathrm{SU}(2)$ is determined by a single number $j$ that can be a positive integer or half-integer: $j=\frac{1}{2}, 1, \frac{3}{2}, 2, \ldots$. The representation Hilbert space is spanned by the orthonormal basis $|j, m\rangle(m=-j,-j+1, \ldots, j-1, j)$.

\subsection{The standard coherent-state basis and related analytic representation}

The standard set of the $\mathrm{SU}(2) \mathrm{CS}$ is obtained for the lowest state $|j,-j\rangle$ chosen as the reference state. The isotropy subgroup $H=\mathrm{U}(1)$ consists of all group elements $h$ of the form $h=\exp \left(i \delta J_{3}\right)$. Thus $h|j,-j\rangle=\exp (-i \delta j)|j,-j\rangle$. The quotient space is $\mathrm{SU}(2) / \mathrm{U}(1)$ (the sphere), and the standard coherent state is specified by a unit vector

$$
\vec{n}=(\sin \theta \cos \varphi, \sin \theta \sin \varphi, \cos \theta)
$$


Then an element $\Omega$ of the quotient space can be written as

$$
\Omega \equiv D(\xi)=\exp \left(\xi J_{+}-\xi^{*} J_{-}\right)
$$

where $\xi=-(\theta / 2) e^{-i \varphi}$. The standard $\mathrm{SU}(2) \mathrm{CS}$ are given by

$$
|j, \zeta\rangle=D(\xi)|j,-j\rangle=\exp \left(\xi J_{+}-\xi^{*} J_{-}\right)|j,-j\rangle=\left(1+|\zeta|^{2}\right)^{-j} \exp \left(\zeta J_{+}\right)|j,-j\rangle,
$$

where $\zeta=(\xi /|\xi|) \tan |\xi|=-\tan (\theta / 2) e^{-i \varphi}$. The parameter $\zeta$ can acquire any complex value. The expansion of the $|j, \zeta\rangle$ states in the orthonormal basis is

$$
|j, \zeta\rangle=\left(1+|\zeta|^{2}\right)^{-j} \sum_{m=-j}^{j}\left[\frac{(2 j) !}{(j+m) !(j-m) !}\right]^{1 / 2} \zeta^{j+m}|j, m\rangle .
$$

The SU(2) CS are normalized but they are not orthogonal to each other:

$$
\left\langle j, \zeta_{1} \mid j, \zeta_{2}\right\rangle=\left(1+\left|\zeta_{1}\right|^{2}\right)^{-j}\left(1+\left|\zeta_{2}\right|^{2}\right)^{-j}\left(1+\zeta_{1}^{*} \zeta_{2}\right)^{2 j}
$$

The identity resolution is

$$
\int d \mu(j, \zeta)|j, \zeta\rangle\langle j, \zeta|=I, \quad d \mu(j, \zeta)=\frac{2 j+1}{\pi} \frac{d^{2} \zeta}{\left(1+|\zeta|^{2}\right)^{2}}
$$

For any state $|\Psi\rangle=\sum_{m=-j}^{j} c_{m}|j, m\rangle$ in the Hilbert space, one can construct the analytic function

$$
f(\zeta)=\left(1+|\zeta|^{2}\right)^{j}\left\langle j, \zeta^{*} \mid \Psi\right\rangle=\sum_{m=-j}^{j} c_{m}\left[\frac{(2 j) !}{(j+m) !(j-m) !}\right]^{1 / 2} \zeta^{j+m} .
$$

Then the state $|\Psi\rangle$ can be expanded in the standard coherent-state basis:

$$
\begin{gathered}
|\Psi\rangle=\int d \mu(j, \zeta)\left(1+|\zeta|^{2}\right)^{-j} f\left(\zeta^{*}\right)|j, \zeta\rangle \\
\langle\Psi \mid \Psi\rangle=\int d \mu(j, \zeta)\left(1+|\zeta|^{2}\right)^{-2 j}\left|f\left(\zeta^{*}\right)\right|^{2}<\infty .
\end{gathered}
$$

The coherent state $\left|j, \zeta_{0}\right\rangle$ is represented by the function

$$
\mathcal{F}\left(j, \zeta_{0} ; \zeta\right)=\left(1+|\zeta|^{2}\right)^{j}\left\langle j, \zeta^{*} \mid j, \zeta_{0}\right\rangle=\left(1+\left|\zeta_{0}\right|^{2}\right)^{-j}\left(1+\zeta_{0} \zeta\right)^{2 j}
$$

The operators $J_{ \pm}$and $J_{3}$ act in the Hilbert space of analytic functions $f(\zeta)$ as first-order differential operators

$$
J_{+}=-\zeta^{2} \frac{d}{d \zeta}+2 j \zeta, \quad J_{-}=\frac{d}{d \zeta}, \quad J_{3}=\zeta \frac{d}{d \zeta}-j
$$

\subsection{The general case}

The eigenvalue equation for the $\mathrm{SU}(2)$ AES is

$$
(\vec{\beta} \cdot \vec{J})|j, \lambda, \vec{\beta}\rangle=\left(\beta_{1} J_{1}+\beta_{2} J_{2}+\beta_{3} J_{3}\right)|j, \lambda, \vec{\beta}\rangle=\lambda|j, \lambda, \vec{\beta}\rangle .
$$

By introducing the analytic function

$$
\Lambda(j, \lambda, \vec{\beta} ; \zeta)=\left(1+|\zeta|^{2}\right)^{j}\left\langle j, \zeta^{*} \mid j, \lambda, \vec{\beta}\right\rangle
$$


we derive the differential equation

$$
\left[\beta_{+}+\beta_{3} \zeta-\beta_{-} \zeta^{2}\right] \frac{d}{d \zeta} \Lambda(j, \lambda, \vec{\beta} ; \zeta)+\left[2 j \beta_{-} \zeta-j \beta_{3}-\lambda\right] \Lambda(j, \lambda, \vec{\beta} ; \zeta)=0,
$$

where we have defined $\beta_{ \pm}=\left(\beta_{1} \pm i \beta_{2}\right) / 2$. Let us also define

$$
b=\sqrt{\beta_{1}^{2}+\beta_{2}^{2}+\beta_{3}^{2}} .
$$

Admissible values of $\vec{\beta}$ and $\lambda$ are determined by the requirement that the function $\Lambda(j, \lambda, \vec{\beta} ; \zeta)$ must be a polynomial of the form (3.9), i.e., it should be normalizable and analytic in the whole $\zeta$ plane. We will see that for any choice of $\vec{\beta}$, there exists at least one such a solution of equation (3.16), i.e., each algebra element $(\vec{\beta} \cdot \vec{J})$ has at least one eigenstate in the Hilbert space of any irreducible representation of $\mathrm{SU}(2)$. In the general case $b \neq 0$, each algebra element has $2 j+1$ eigenstates with symmetric spectrum: $\lambda=-j b,(-j+1) b, \ldots,(j-1) b, j b$. In the degenerate case $b=0$, each algebra element has only one eigenstate with eigenvalue $\lambda=0$. All these degenerate eigenstates are the standard CS.

We start by considering the general case $b \neq 0$. For $\beta_{+} \neq 0$, the solution of equation (3.16) reads

$$
\Lambda(j, \lambda, \vec{\beta} ; \zeta)=\mathcal{N}^{-1 / 2}\left(1-\tau_{-} \zeta\right)^{j+m_{0}}\left(1-\tau_{+} \zeta\right)^{j-m_{0}}
$$

where $\mathcal{N}$ is a normalization factor, and we use the following notation:

$$
\begin{aligned}
& \tau_{ \pm}=\left(\beta_{1}-i \beta_{2}\right) /\left(\beta_{3} \pm b\right), \\
& m_{0}=\lambda / b .
\end{aligned}
$$

The condition of the analyticity for the function $\Lambda(\zeta)$ requires that $m_{0}$ can take only the values:

$$
m_{0}=-j,-j+1, \ldots, j-1, j .
$$

This condition means that the $\mathrm{SU}(2)$ AES have the discrete spectrum $\lambda=m_{0} b$.

We can compare the function $\Lambda(j, \lambda, \vec{\beta} ; \zeta)$ of equation (3.18) with the function $\mathcal{F}\left(j, \zeta_{0} ; \zeta\right)$ of equation (3.12) that represents the standard coherent state $\left|j, \zeta_{0}\right\rangle$. We find that the algebra eigenstate $|j, \lambda, \vec{\beta}\rangle$ belongs to the standard set of the CS when $m_{0}= \pm j$. Then $\zeta_{0}=-\tau_{\mp}$, respectively. The normalization factor in this case is identified as $\mathcal{N}=\left(1+\left|\zeta_{0}\right|^{2}\right)^{2 j}$. For example, we can choose $\vec{\beta}$ to be a unit vector $\vec{\beta}=\vec{n}=(\sin \theta \cos \varphi, \sin \theta \sin \varphi, \cos \theta)$, and $m_{0}=-j$. Then

$$
\zeta_{0}=-\frac{\sin \theta e^{-i \varphi}}{\cos \theta+1}=-\tan (\theta / 2) e^{-i \varphi} .
$$

It means that the standard CS form a subset of the AES with the corresponding eigenvalue equation

$$
\left[(\sin \theta \cos \varphi) J_{1}+(\sin \theta \sin \varphi) J_{2}+(\cos \theta) J_{3}\right]\left|j, \zeta_{0}\right\rangle=-j\left|j, \zeta_{0}\right\rangle .
$$

This result can be found by acting with $D\left(\xi_{0}\right)$ on both sides of equation $J_{3}|j,-j\rangle=-j|j,-j\rangle$.

\subsection{The expansion in the orthonormal basis and quantum statistics}

The decomposition of the AES $|j, \lambda, \vec{\beta}\rangle$ over the orthonormal basis can be obtained by expanding the function $\Lambda(j, \lambda, \vec{\beta} ; \zeta)$ of equation (3.18) into a power series in $\zeta$. This can be done by using the generating function for the Lagrange polynomials $g_{n}^{(\alpha, \beta)}(u, v)$ (Erdélyi et al., 1953, Vol. 3, Sec. 19.11; Srivastava and Manocha, 1984, Secs. 1.11, 8.5):

$$
(1-u \zeta)^{-\alpha}(1-v \zeta)^{-\beta}=\sum_{n=0}^{\infty} g_{n}^{(\alpha, \beta)}(u, v) \zeta^{n}
$$


The Lagrange polynomials are related to the more familiar Jacobi polynomials $P_{n}^{(\alpha, \beta)}(x)$ via the relation (Srivastava and Manocha, 1984, Sec. 8.5)

$$
g_{n}^{(\alpha, \beta)}(u, v)=(v-u)^{n} P_{n}^{(-\alpha-n,-\beta-n)}\left(\frac{u+v}{u-v}\right) .
$$

Thus one obtains the following generating function for the Jacobi polynomials:

$$
(1-u \zeta)^{\mu}(1-v \zeta)^{\nu}=\sum_{n=0}^{\infty}(v-u)^{n} P_{n}^{(\mu-n, \nu-n)}\left(\frac{u+v}{u-v}\right) \zeta^{n} .
$$

Using this expression, we obtain the power series for the function $\Lambda(j, \lambda, \vec{\beta} ; \zeta)$ of equation (3.18):

$$
\Lambda(j, \lambda, \vec{\beta} ; \zeta)=\mathcal{N}^{-1 / 2} \sum_{m=-j}^{j} P_{j+m}^{\left(m_{0}-m,-m_{0}-m\right)}(x)(\kappa \zeta)^{j+m},
$$

where we have defined

$$
\begin{aligned}
& \kappa=\tau_{+}-\tau_{-}=2 b /\left(\beta_{1}+i \beta_{2}\right), \\
& x=\left(\tau_{-}+\tau_{+}\right) /\left(\tau_{-}-\tau_{+}\right)=\beta_{3} / b .
\end{aligned}
$$

The series 3.27 is finite due to the fact that

$$
P_{n}^{\left(j+m_{0}-n, j-m_{0}-n\right)}(x)=0 \quad \text { for } n>2 j .
$$

Comparing the expansion (3.27) with the general formula (3.9), we find the decomposition of the AES over the orthonormal basis:

$$
|j, \lambda, \vec{\beta}\rangle=\mathcal{N}^{-1 / 2} \sum_{m=-j}^{j}\left[\frac{(j+m) !(j-m) !}{(2 j) !}\right]^{1 / 2} P_{j+m}^{\left(m_{0}-m,-m_{0}-m\right)}(x) \kappa^{j+m}|j, m\rangle .
$$

The normalization factor is given by

$$
\mathcal{N}=\sum_{n=0}^{2 j} \frac{n !(2 j-n) !}{(2 j) !}\left|P_{n}^{\left(j+m_{0}-n, j-m_{0}-n\right)}(x)\right|^{2} t^{n},
$$

where $t=|\kappa|^{2}$. The summation in (3.32) can be formally continued up to infinity because all the terms with $n>2 j$ vanish. Then we can use the summation theorem for the Jacobi polynomials (Srivastava and Manocha, 1984, Sec. 2.3, Eqs. 60, 62), that can be written in the form

$$
\sum_{n=0}^{\infty} \frac{n ! \Gamma(\mu+\nu+1-n)}{\Gamma(\mu+\nu+1)}\left|P_{n}^{(\mu-n, \nu-n)}(x)\right|^{2} t^{n}=S_{+}^{\mu} S_{-}^{\nu} F\left(-\nu,-\mu ;-\mu-\nu ; \frac{t}{S_{+} S_{-}}\right)
$$

for $\mu+\nu \geq 0$. Here, $F(a, b ; c ; z)$ is the hypergeometric function, and we have defined

$$
S_{ \pm}=1+|x \pm 1|^{2} t / 4=1+\left|\tau_{\mp}\right|^{2} .
$$

If $\mu$ and $\nu$ are nonnegative integers, we can use the relation between the hypergeometric function and the Jacobi polynomials (Erdélyi et al., 1953, Vol. 2, Sec. 10.8), that can be expressed in the form

$$
F(-\mu,-\nu ;-\mu-\nu ; z)=(-1)^{n} \frac{\mu ! \nu !}{(\mu+\nu) !} P_{n}^{(-\mu-\nu-1,0)}(1-2 z), \quad n=\min (\mu, \nu)
$$


Therefore, we obtain the closed expression for the normalization factor:

$$
\mathcal{N}=(-1)^{j-\left|m_{0}\right|} S_{+}^{j+m_{0}} S_{-}^{j-m_{0}} \frac{\left(j+m_{0}\right) !\left(j-m_{0}\right) !}{(2 j) !} P_{j-\left|m_{0}\right|}^{(-2 j-1,0)}\left(1-\frac{2 t}{S_{+} S_{-}}\right) .
$$

It can be easily verified that for $m_{0}= \pm j$, these formulas reduce to the corresponding results for the standard coherent state $\left|j, \zeta_{0}\right\rangle$ with $\zeta_{0}=-\tau_{\mp}$, respectively.

The above analytic expressions can be used for calculations of quantum statistical properties of the $\mathrm{SU}(2)$ AES. We demonstrate how such a calculation can be performed by considering moments of the generator $J_{3}$. By using the property $J_{3}|j, m\rangle=m|j, m\rangle$ and formula (3.32), we can express moments of $J_{3}$ over the AES as derivatives of $\mathcal{N}$ with respect to $t$ :

$$
\begin{gathered}
\left\langle J_{3}\right\rangle=\frac{t}{\mathcal{N}} \frac{\partial \mathcal{N}}{\partial t}-j \\
\left(\Delta J_{3}\right)^{2}=\frac{t^{2}}{\mathcal{N}} \frac{\partial^{2} \mathcal{N}}{\partial t^{2}}+\frac{t}{\mathcal{N}} \frac{\partial \mathcal{N}}{\partial t}-\left(\frac{t}{\mathcal{N}} \frac{\partial \mathcal{N}}{\partial t}\right)^{2} .
\end{gathered}
$$

By using the formula (Erdélyi et al., 1953, Vol. 2, Sec. 10.8)

$$
\frac{d P_{n}^{(\mu, \nu)}(x)}{d x}=\frac{n+\mu+\nu+1}{2} P_{n-1}^{(\mu+1, \nu+1)}(x)
$$

and the differential equation for the Jacobi polynomials, we obtain exact analytic expressions for the moments of $J_{3}$ :

$$
\begin{gathered}
\left\langle J_{3}\right\rangle=\frac{j Y+m_{0}\left(S_{+}-S_{-}\right)}{S_{+} S_{-}}-\frac{\left(j+\left|m_{0}\right|\right) Y t}{S_{+}^{2} S_{-}^{2}} \Omega, \\
\left(\Delta J_{3}\right)^{2}=\left(j+m_{0}\right) \frac{S_{+}-1}{S_{+}^{2}}+\left(j-m_{0}\right) \frac{S_{-}-1}{S_{-}^{2}}+\frac{\left(j^{2}-m_{0}^{2}\right) Y^{2} t}{\left(S_{+} S_{-}-t\right) S_{+}^{2} S_{-}^{2}} \\
+\frac{\left(j+\left|m_{0}\right|\right) t}{S_{+}^{3} S_{-}^{3}}\left(\frac{S_{+} S_{-} Y^{2}}{S_{+} S_{-}-t}+2 j Y^{2}+Z\right) \Omega-\frac{\left(j+\left|m_{0}\right|\right)^{2} Y^{2} t^{2}}{S_{+}^{4} S_{-}^{4}} \Omega^{2} .
\end{gathered}
$$

Here, we have introduced the following notation:

$$
\begin{gathered}
Y=S_{+} S_{-}-S_{+}-S_{-}, \\
Z=S_{+}^{2}\left(1-S_{-}\right)+S_{-}^{2}\left(1-S_{+}\right), \\
\Omega=\left[P_{j-\left|m_{0}\right|}^{(-2 j-1,0)}\left(1-\frac{2 t}{S_{+} S_{-}}\right)\right]^{-1} P_{j-\left|m_{0}\right|-1}^{(-2 j, 1)}\left(1-\frac{2 t}{S_{+} S_{-}}\right), \quad\left|m_{0}\right|<j .
\end{gathered}
$$

For $m_{0}= \pm j$, we have $\Omega=0$, and then we recover the known results for the SU(2) CS (Wodkiewicz and Eberly, 1985). The expressions for $\left\langle J_{3}\right\rangle$ and $\left(\Delta J_{3}\right)^{2}$ are significantly simplified in the case $Y=0$, which means

$$
\left|\tau_{+} \tau_{-}\right|=1 \Leftrightarrow\left|\frac{\left(\beta_{1}-i \beta_{2}\right)^{2}}{\beta_{1}^{2}+\beta_{2}^{2}}\right|=1 .
$$

This condition is satisfied in the important case $\beta_{1}=a \beta_{2}$, where $a$ is any real number (this includes the case when $\beta_{1}$ or $\beta_{2}$ vanishes). Then we obtain

$$
\begin{gathered}
\left\langle J_{3}\right\rangle=\frac{h-1}{h+1} m_{0}, \\
\left(\Delta J_{3}\right)^{2}=\frac{2 j h}{(h+1)^{2}}-\frac{2\left(j+\left|m_{0}\right|\right) h^{2} t}{(h+1)^{4}} \Omega
\end{gathered}
$$

where

$$
h=\left|\tau_{-}\right|^{2}=1 /\left|\tau_{+}\right|^{2} .
$$




\subsection{Some special cases}

For $\beta_{+}=0$ and $\beta_{3} \neq 0$, we obtain $\tau_{-} \rightarrow \infty$, so we cannot use formula (3.18). In this case the solution of equation (3.16) is

$$
\Lambda(j, \lambda, \vec{\beta} ; \zeta)=\mathcal{N}^{-1 / 2} \zeta^{j+m_{0}}\left(1-\tau_{+} \zeta\right)^{j-m_{0}},
$$

where $\tau_{+}=\beta_{1} / \beta_{3}$ and $m_{0}=\lambda / \beta_{3}$. The condition of the analyticity requires that $m_{0}$ can take only the discrete values (3.21). The results of section 3.3 cannot be used in this special case, but corresponding expressions can be obtained by expanding the function (3.49) into the power series. We find

$$
\begin{gathered}
|j, \lambda, \vec{\beta}\rangle=\mathcal{N}^{-1 / 2} \sum_{m=m_{0}}^{j}\left[\frac{(j+m) !}{(j-m) !}\right]^{1 / 2} \frac{\left(-\tau_{+}\right)^{m-m_{0}}}{\left(m-m_{0}\right) !}|j, m\rangle, \\
\mathcal{N}=\frac{\left(j+m_{0}\right) !}{\left(j-m_{0}\right) !} P_{j-m_{0}}^{\left(0,2 m_{0}\right)}\left(2\left|\tau_{+}\right|^{2}+1\right) .
\end{gathered}
$$

For $m_{0}=-j$, the function (3.49) represents the standard coherent state $\left|j, \zeta_{0}\right\rangle$ with $\zeta_{0}=-\tau_{+}$. The corresponding eigenvalue equation is

$$
\left(J_{3}-\zeta_{0} J_{+}\right)\left|j, \zeta_{0}\right\rangle=-j\left|j, \zeta_{0}\right\rangle .
$$

For $m_{0}=j$, we find $\Lambda(\zeta)=\zeta^{2 j}$ that represents the state $|j, j\rangle$.

For $\beta_{-}=0$ and $\beta_{3} \neq 0$, we can use the general results of the preceding sections with $b=\beta_{3}$, $\tau_{+}=0$ and $\tau_{-}=-\beta_{3} / \beta_{1}$. This gives $\kappa=-\tau_{-}=\beta_{3} / \beta_{1}, x=1, S_{+}=1+t\left(\right.$ where $\left.t=|\kappa|^{2}\right)$ and $S_{-}=1$. The corresponding analytic function is

$$
\Lambda(j, \lambda, \vec{\beta} ; \zeta)=\mathcal{N}^{-1 / 2}\left(1-\tau_{-} \zeta\right)^{j+m_{0}}
$$

For $m_{0}=j$, this function represents the standard coherent state $\left|j, \zeta_{0}\right\rangle$ with $\zeta_{0}=\beta_{3} / \beta_{1}$. The corresponding eigenvalue equation is

$$
\left(J_{3}+\zeta_{0}^{-1} J_{-}\right)\left|j, \zeta_{0}\right\rangle=j\left|j, \zeta_{0}\right\rangle .
$$

For $m_{0}=-j$, we find $\Lambda(\zeta)=1$ that corresponds to the state $|j,-j\rangle$.

For the degenerate case $b=0$, the solution of equation (3.16) is

$$
\Lambda(j, \lambda, \vec{\beta} ; \zeta)=\mathcal{N}^{-1 / 2}(1-\tau \zeta)^{2 j} \exp \left(-\frac{2 \lambda}{\beta_{3}} \frac{1}{1-\tau \zeta}\right)
$$

where $\tau=2 \beta_{-} / \beta_{3}=-\beta_{3} / 2 \beta_{+}$. This function is analytic only for $\lambda=0$. Then we obtain

$$
\Lambda(j, \lambda=0, \vec{\beta} ; \zeta)=\mathcal{N}^{-1 / 2}(1-\tau \zeta)^{2 j} .
$$

This function represents the standard coherent state $\left|j, \zeta_{0}\right\rangle$ with $\zeta_{0}=-\tau$ and $\mathcal{N}=\left(1+\left|\zeta_{0}\right|^{2}\right)^{2 j}$. For example, we can choose $\vec{\beta}=\left(1-\zeta_{0}^{2},-i\left(1+\zeta_{0}^{2}\right), 2 \zeta_{0}\right)$. Then the standard CS satisfy the eigenvalue equation

$$
\left(J_{-}+2 \zeta_{0} J_{3}-\zeta_{0}^{2} J_{+}\right)\left|j, \zeta_{0}\right\rangle=0 .
$$

For $\beta_{-}=\beta_{3}=0$, the only normalizable solution of equation (3.16) is $\Lambda(\zeta)=1$ that represents the state $|j,-j\rangle$ (that is the standard coherent state with $\zeta_{0}=0$ ). Analogously, for $\beta_{+}=\beta_{3}=0$, the corresponding function is $\Lambda(\zeta)=\zeta^{2 j}$ that represents the state $|j, j\rangle$ (that is the standard coherent state with $\left.\zeta_{0} \rightarrow \infty\right)$. 


\subsection{The $\mathrm{SU}(2)$ intelligent states}

The SU(2) generalized IS were defined by Trifonov (1994) as the eigenstates of the operator $\eta J_{1}-i J_{2}$ [see equation (2.15)]. In our notation, the generalized IS are the AES with $\vec{\beta}=(\eta,-i, 0)$. For $\eta^{2} \neq 1$, the corresponding analytic function is given by the particular case of equation (3.18) with $b=\sqrt{\eta^{2}-1}$ and

$$
\tau_{ \pm}= \pm \sqrt{\frac{\eta-1}{\eta+1}} .
$$

All the results of section 3.3 are valid here with $\kappa=2 \tau_{+}, x=0$, and $S_{+}=S_{-}=1+\left|\tau_{+}\right|^{2}$. The generalized intelligent state is also the standard coherent state when $m_{0}= \pm j$. The corresponding coherent-state amplitude is $\zeta_{0}=-\tau_{\mp}$, respectively. Since $\eta$ is an arbitrary complex number (excluding \pm 1 ), $\zeta_{0}$ also can acquire any complex value (excluding 0 and $\infty$ ). For $\eta=1$, the generalized IS are reduced to the state $|j,-j\rangle$ that is the standard coherent state with $\zeta_{0}=0$. For $\eta=-1$, the generalized IS are reduced to the state $|j, j\rangle$ that is the standard coherent state with $\zeta_{0} \rightarrow \infty$. Hence, the standard set of the $\mathrm{SU}(2)$ CS is a subset of the $\mathrm{SU}(2)$ generalized IS.

We also discuss briefly three types of the SU(2) ordinary IS. The $J_{1}-J_{2}$ IS are determined, according to equation (2.16), as the eigenstates of the operator $J_{1}+i \gamma J_{2}$, where $\gamma$ is a real parameter. These states are the $\operatorname{SU}(2) \operatorname{AES}$ with $\vec{\beta}=(1, i \gamma, 0)$. For $\gamma^{2} \neq 1$, the corresponding analytic function is given by the particular case of equation (3.18) with $b=\sqrt{1-\gamma^{2}}$ and

$$
\tau_{ \pm}= \pm \sqrt{\frac{1+\gamma}{1-\gamma}}
$$

All the results of section 3.3 are valid here with $\kappa=2 \tau_{+}, x=0$, and $S_{+}=S_{-}=1+\left|\tau_{+}\right|^{2}$. The $J_{1}-J_{2}$ IS coincide with the standard CS for $m_{0}= \pm j$. Since $\gamma$ is real, the corresponding coherent-state amplitude $\zeta_{0}$ is real for $|\gamma|<1$ and pure imaginary for $|\gamma|>1$. Therefore the set of the $J_{1}-J_{2}$ IS and the standard set of the $\mathrm{SU}(2)$ CS have an intersection.

The $J_{1}-J_{3}$ IS are determined, according to equation (2.16), as the eigenstates of the operator $J_{1}+i \gamma J_{3}$. These states are the SU(2) AES with $\vec{\beta}=(1,0, i \gamma)$. For $\gamma^{2} \neq 1$, the corresponding analytic function is given by the particular case of equation (3.18) with $b=\sqrt{1-\gamma^{2}}$ and

$$
\tau_{ \pm}=\left(i \gamma \pm \sqrt{1-\gamma^{2}}\right)^{-1}
$$

All the results of section 3.3 are valid here with $\kappa=2 \sqrt{1-\gamma^{2}}, x=i \gamma / \sqrt{1-\gamma^{2}}$. Note that $\left|\tau_{+} \tau_{-}\right|=1$, and therefore we can use simple expressions (3.46) and (3.47). For $\gamma^{2}<1$, we have $S_{+}=S_{-}=2$ and $h=1$. For $\gamma^{2}>1$, we obtain $h=2 \gamma^{2}+2 \gamma \sqrt{\gamma^{2}-1}-1$. The intersection between the $J_{1}-J_{3}$ IS and the standard CS is obtained for $m_{0}= \pm j$. In the case $\gamma= \pm 1$, we have $\lambda=b=0$, and then the corresponding analytic function is $\Lambda(\zeta)=\mathcal{N}^{-1 / 2}(1 \pm i \zeta)^{2 j}$. This function corresponds to the standard coherent state $\left|j, \zeta_{0}\right\rangle$ with $\zeta_{0}= \pm i$.

The $J_{2^{-}} J_{3}$ IS are determined, according to equation (2.16), as the eigenstates of the operator $J_{2}+i \gamma J_{3}$. These states are the $\mathrm{SU}(2)$ AES with $\vec{\beta}=(0,1, i \gamma)$. For $\gamma^{2} \neq 1$, the corresponding analytic function is given by the particular case of equation (3.18) with $b=\sqrt{1-\gamma^{2}}$ and

$$
\tau_{ \pm}=\left(-\gamma \pm i \sqrt{1-\gamma^{2}}\right)^{-1}
$$

All the results of section 3.3 are valid here with $\kappa=-2 i \sqrt{1-\gamma^{2}}, x=i \gamma / \sqrt{1-\gamma^{2}}$. Here $\left|\tau_{+} \tau_{-}\right|=1$, and therefore we can use simple expressions (3.46) and (3.47). For $\gamma^{2}<1$, we find $S_{+}=S_{-}=2$ and $h=1$. For $\gamma^{2}>1$, we obtain $h=2 \gamma^{2}+2 \gamma \sqrt{\gamma^{2}-1}-1$. The intersection between the $J_{1}-J_{3}$ IS and the standard CS is obtained for $m_{0}= \pm j$. In the case $\gamma= \pm 1$, we have $\lambda=b=0$, and then the corresponding analytic function is $\Lambda(\zeta)=\mathcal{N}^{-1 / 2}(1 \pm \zeta)^{2 j}$. This function corresponds to the standard coherent state $\left|j, \zeta_{0}\right\rangle$ with $\zeta_{0}= \pm 1$. 


\section{THE SU(1,1) ALGEBRA EIGENSTATES}

In this section we consider the AES for the $\mathrm{SU}(1,1)$ group which is the most elementary noncompact non-Abelian simple Lie group. It has several series of unitary irreducible representations: discrete, continuous and supplementary (Bargmann, 1947; Vilenkin, 1968). In the present work we discuss only the case of the discrete series which has many well-known physical applications (Perelomov, 1986). The Lie algebra corresponding to the group $\mathrm{SU}(1,1)$ is spanned by the three operators $\left\{K_{1}, K_{2}, K_{3}\right\}$,

$$
\left[K_{1}, K_{2}\right]=-i K_{3}, \quad\left[K_{2}, K_{3}\right]=i K_{1}, \quad\left[K_{3}, K_{1}\right]=i K_{2} .
$$

It is convenient to use raising and lowering operators $K_{ \pm}=K_{1} \pm i K_{2}$ which satisfy the following commutation relations:

$$
\left[K_{3}, K_{ \pm}\right]= \pm K_{ \pm}, \quad\left[K_{-}, K_{+}\right]=2 K_{3} .
$$

The Casimir operator $K^{2}=K_{3}^{2}-K_{1}^{2}-K_{2}^{2}$ for any unitary irreducible representation is the identity operator times a number: $K^{2}=k(k-1) I$. Representations of SU $(1,1)$ are determined by a single number $k$; for the discrete-series representations this number acquires discrete values $k=\frac{1}{2}, 1, \frac{3}{2}, 2, \ldots$. The representation Hilbert space is spanned by the orthonormal basis $|k, n\rangle$ $(n=0,1,2, \ldots)$.

The SU(1,1) AES can be investigated by using two alternative analytic representations: one of them is based on the standard CS (Perelomov, 1986) and the other is based on the so-called Barut-Girardello (BG) states (Barut and Girardello, 1971).

\subsection{The standard coherent-state basis and the analytic representation in the unit disk}

The standard set of the $\mathrm{SU}(1,1) \mathrm{CS}$ is obtained for the lowest state $|k, 0\rangle$ chosen as the reference state. The isotropy subgroup $H=\mathrm{U}(1)$ consists of all group elements $h$ of the form $h=\exp \left(i \delta K_{3}\right)$. Thus $h|k, 0\rangle=\exp (i \delta k)|k, 0\rangle$. The quotient space is $\mathrm{SU}(1,1) / \mathrm{U}(1)$ (the upper sheet of the twosheet hyperboloid), and the standard coherent state is specified by a unit pseudo-Euclidean vector

$$
\vec{n}=(\sinh \chi \cos \varphi, \sinh \chi \sin \varphi, \cosh \chi) .
$$

Then an element $\Omega$ of the quotient space can be written as

$$
\Omega \equiv D(\xi)=\exp \left(\xi K_{+}-\xi^{*} K_{-}\right),
$$

where $\xi=-(\chi / 2) e^{-i \varphi}$. The standard $\mathrm{SU}(1,1) \mathrm{CS}$ are given by

$$
|k, \zeta\rangle=D(\xi)|k, 0\rangle=\exp \left(\xi K_{+}-\xi^{*} K_{-}\right)|k, 0\rangle=\left(1-|\zeta|^{2}\right)^{k} \exp \left(\zeta K_{+}\right)|k, 0\rangle,
$$

where $\zeta=(\xi /|\xi|) \tanh |\xi|=-\tanh (\chi / 2) e^{-i \varphi}$. The parameter $\zeta$ is restricted by $|\zeta|<1$. The expansion of the $|k, \zeta\rangle$ states in the orthonormal basis is

$$
|k, \zeta\rangle=\left(1-|\zeta|^{2}\right)^{k} \sum_{n=0}^{\infty}\left[\frac{\Gamma(2 k+n)}{n ! \Gamma(2 k)}\right]^{1 / 2} \zeta^{n}|k, n\rangle .
$$

The SU(1,1) CS are normalized but they are not orthogonal to each other:

$$
\left\langle k, \zeta_{1} \mid k, \zeta_{2}\right\rangle=\left(1-\left|\zeta_{1}\right|^{2}\right)^{k}\left(1-\left|\zeta_{2}\right|^{2}\right)^{k}\left(1-\zeta_{1}^{*} \zeta_{2}\right)^{-2 k} .
$$

The identity resolution is (for $k>\frac{1}{2}$ )

$$
\int d \mu(k, \zeta)|k, \zeta\rangle\langle k, \zeta|=I, \quad d \mu(k, \zeta)=\frac{2 k-1}{\pi} \frac{d^{2} \zeta}{\left(1-|\zeta|^{2}\right)^{2}}
$$


and for $k=\frac{1}{2}$ the limit $k \rightarrow \frac{1}{2}$ must be taken after the integration is carried out in the general form. For any state $|\Psi\rangle=\sum_{n=0}^{\infty} c_{n}|k, n\rangle$ in the Hilbert space, one can construct the analytic function

$$
f(\zeta)=\left(1-|\zeta|^{2}\right)^{-k}\left\langle k, \zeta^{*} \mid \Psi\right\rangle=\sum_{n=0}^{\infty} c_{n}\left[\frac{\Gamma(2 k+n)}{n ! \Gamma(2 k)}\right]^{1 / 2} \zeta^{n} .
$$

Since $|\zeta|<1$, this analytic representation is referred to as the representation in the unit disk. The expansion of the state $|\Psi\rangle$ in the standard coherent-state basis is given by

$$
\begin{gathered}
|\Psi\rangle=\int d \mu(k, \zeta)\left(1-|\zeta|^{2}\right)^{k} f\left(\zeta^{*}\right)|k, \zeta\rangle, \\
\langle\Psi \mid \Psi\rangle=\int d \mu(k, \zeta)\left(1-|\zeta|^{2}\right)^{2 k}\left|f\left(\zeta^{*}\right)\right|^{2}<\infty .
\end{gathered}
$$

The coherent state $\left|k, \zeta_{0}\right\rangle$ is represented by the function

$$
\mathcal{F}\left(k, \zeta_{0} ; \zeta\right)=\left(1-|\zeta|^{2}\right)^{-k}\left\langle k, \zeta^{*} \mid k, \zeta_{0}\right\rangle=\left(1-\left|\zeta_{0}\right|^{2}\right)^{k}\left(1-\zeta_{0} \zeta\right)^{-2 k}
$$

The operators $K_{ \pm}$and $K_{3}$ act in the Hilbert space of analytic functions $f(\zeta)$ as first-order differential operators

$$
K_{+}=\zeta^{2} \frac{d}{d \zeta}+2 k \zeta, \quad K_{-}=\frac{d}{d \zeta}, \quad K_{3}=\zeta \frac{d}{d \zeta}+k
$$

\subsection{The general case}

The eigenvalue equation for the $\mathrm{SU}(1,1)$ AES is

$$
(\vec{\beta} \cdot \vec{K})|k, \lambda, \vec{\beta}\rangle=\left(\beta_{1} K_{1}+\beta_{2} K_{2}+\beta_{3} K_{3}\right)|k, \lambda, \vec{\beta}\rangle=\lambda|k, \lambda, \vec{\beta}\rangle .
$$

Some particular cases of this equation were considered by Barut and Girardello (1971), Lindblad and Nagel (1970), Solomon (1971) and Nagel (1995). By introducing the analytic function

$$
\Lambda(k, \lambda, \vec{\beta} ; \zeta)=\left(1-|\zeta|^{2}\right)^{-k}\left\langle k, \zeta^{*} \mid k, \lambda, \vec{\beta}\right\rangle,
$$

we derive the differential equation

$$
\left[\beta_{+}+\beta_{3} \zeta+\beta_{-} \zeta^{2}\right] \frac{d}{d \zeta} \Lambda(k, \lambda, \vec{\beta} ; \zeta)+\left[2 k \beta_{-} \zeta+k \beta_{3}-\lambda\right] \Lambda(k, \lambda, \vec{\beta} ; \zeta)=0
$$

where we have defined $\beta_{ \pm}=\left(\beta_{1} \pm i \beta_{2}\right) / 2$. Let us also define

$$
b=\sqrt{\beta_{3}^{2}-\beta_{1}^{2}-\beta_{2}^{2}} .
$$

Admissible values of $\vec{\beta}$ and $\lambda$ are determined by the requirement that the function $\Lambda(k, \lambda, \vec{\beta} ; \zeta)$ must be analytic in the unit disk. We will see that the non-compactness of the $\mathrm{SU}(1,1)$ group leads to a reach structure that is absent in the $\mathrm{SU}(2)$ case. In the general case $b \neq 0$, there are three classes of algebra elements $(\vec{\beta} \cdot \vec{K})$. The first class consists of elements with a continuous spectrum (no restrictions on $\lambda$ ). Elements in the second class have a discrete equidistant spectrum: in one subclass $\lambda=(k+l) b$ and in the other subclass $\lambda=-(k+l) b$ (where $l=0,1,2, \ldots)$. The third class includes elements that have not any normalizable eigenstate. In the degenerate case $b=0$, there are two classes of algebra elements. The first class consists of elements with a continuous spectrum (no restrictions on $\lambda$ ), while the second class includes elements that have not any normalizable eigenstate. In the degenerate case there are no algebra elements with a discrete spectrum. 
We first consider the general case $b \neq 0$. For $\beta_{+} \neq 0$, the solution of equation (4.16) is

$$
\Lambda(k, \lambda, \vec{\beta} ; \zeta)=\mathcal{N}^{-1 / 2}\left(1+\tau_{-} \zeta\right)^{-k+r}\left(1+\tau_{+} \zeta\right)^{-k-r},
$$

where $\mathcal{N}$ is a normalization factor, and we use the following notation:

$$
\begin{aligned}
& \tau_{ \pm}=\left(\beta_{1}-i \beta_{2}\right) /\left(\beta_{3} \pm b\right) \\
& r=\lambda / b .
\end{aligned}
$$

Now we analyse the analyticity condition for the function $\Lambda(\zeta)$ of equation (4.18). If $\left|\tau_{+}\right|<1$ and $\left|\tau_{-}\right|<1$, then there are no restrictions on $\lambda$ (i.e., the corresponding algebra elements have a continuous complex spectrum). If $\left|\tau_{+}\right|<1$ and $\left|\tau_{-}\right| \geq 1$, then the analyticity condition requires $r=k+l$ (where $l=0,1,2, \ldots$ ), i.e., the spectrum is discrete and equidistant:

$$
\lambda=(k+l) b .
$$

If $\left|\tau_{+}\right| \geq 1$ and $\left|\tau_{-}\right|<1$, then the analyticity condition requires $r=-(k+l)$, and once again the spectrum is discrete and equidistant:

$$
\lambda=-(k+l) b .
$$

If $\left|\tau_{+}\right| \geq 1$ and $\left|\tau_{-}\right| \geq 1$, then the function $\Lambda(\zeta)$ of equation (4.18) cannot be analytic in the unit disk for any value of $\lambda$. This region in the parameter space is forbidden, i.e., the corresponding algebra elements have no normalizable eigenstates. Note that there are algebra elements whose eigenstates are unnormalizable in sense of equation (4.11), but these states can be orthonormalized by the delta function. For example, such generalized orthonormality relations exist for the eigenstates of the operators $K_{1}$ and $K_{2}$, for which $\left|\tau_{+}\right|=\left|\tau_{-}\right|=1$ (Lindblad and Nagel, 1970; Nagel, 1995). In our notation, these operators formally belong to the forbidden region of the parameter space. The structure of the parameter space is described in Fig. 1.

Figure 1: The structure of the parameter space for the SU(1,1) AES in the general case $b \neq 0$.

We can compare the function $\Lambda(k, \lambda, \vec{\beta} ; \zeta)$ of equation (4.18) with the function $\mathcal{F}\left(k, \zeta_{0} ; \zeta\right)$ of equation (4.12) that represents the standard coherent state $\left|k, \zeta_{0}\right\rangle$. We find that the algebra eigenstate $|k, \lambda, \vec{\beta}\rangle$ belongs to the standard set of the CS when $r= \pm k$, i.e., $\lambda= \pm k b$. Then $\zeta_{0}=-\tau_{ \pm}$, respectively. The condition $\left|\zeta_{0}\right|<1$ is equivalent to the analyticity condition: $\left|\tau_{ \pm}\right|<1$ for $r= \pm k$, respectively. The normalization factor $\mathcal{N}$ in this case is identified as $\mathcal{N}=\left(1-\left|\zeta_{0}\right|^{2}\right)^{-2 k}$. For example, we can choose $\vec{\beta}$ to be a unit pseudo-Euclidean vector $\vec{\beta}=\vec{n}=(\sinh \chi \cos \varphi, \sinh \chi \sin \varphi, \cosh \chi)$, and $r=k$. Then

$$
\zeta_{0}=-\frac{\sinh \chi e^{-i \varphi}}{\cosh \chi+1}=-\tanh (\chi / 2) e^{-i \varphi} .
$$

It means that the standard CS form a subset of the AES with the corresponding eigenvalue equation

$$
\left[(\sinh \chi \cos \varphi) K_{1}+(\sinh \chi \sin \varphi) K_{2}+(\cosh \chi) K_{3}\right]\left|k, \zeta_{0}\right\rangle=k\left|k, \zeta_{0}\right\rangle .
$$

This result can be found by acting with $D\left(\xi_{0}\right)$ on both sides of equation $K_{3}|k, 0\rangle=k|k, 0\rangle$.

\subsection{The expansion in the orthonormal basis and quantum statistics}

In the allowed region of the parameter space, we can use equation (3.26) for expanding the function $\Lambda(k, \lambda, \vec{\beta} ; \zeta)$ of equation (4.18) into the power series:

$$
\Lambda(k, \lambda, \vec{\beta} ; \zeta)=\mathcal{N}^{-1 / 2} \sum_{n=0}^{\infty} P_{n}^{(-k+r-n,-k-r-n)}(x)(\kappa \zeta)^{n}
$$


where we have defined

$$
\begin{aligned}
& \kappa=\tau_{+}-\tau_{-}=-2 b /\left(\beta_{1}+i \beta_{2}\right), \\
& x=\left(\tau_{-}+\tau_{+}\right) /\left(\tau_{-}-\tau_{+}\right)=\beta_{3} / b .
\end{aligned}
$$

Comparing the expansion (4.25) with the general formula (4.9), we find the decomposition of the AES over the orthonormal basis:

$$
|k, \lambda, \vec{\beta}\rangle=\mathcal{N}^{-1 / 2} \sum_{n=0}^{\infty}\left[\frac{n ! \Gamma(2 k)}{\Gamma(2 k+n)}\right]^{1 / 2} P_{n}^{(-k+r-n,-k-r-n)}(x) \kappa^{n}|k, n\rangle .
$$

The normalization factor is given by

$$
\mathcal{N}=\sum_{n=0}^{\infty} \frac{n ! \Gamma(2 k)}{\Gamma(2 k+n)}\left|P_{n}^{(-k+r-n,-k-r-n)}(x)\right|^{2} t^{n}
$$

where $t=|\kappa|^{2}$. The summation theorem for the Jacobi polynomials (Srivastava and Manocha, 1984, Sec. 2.3, Eqs. 60, 62) can be written in the form

$$
\sum_{n=0}^{\infty} \frac{n ! \Gamma(\mu+\nu)}{\Gamma(\mu+\nu+n)}\left|P_{n}^{(-\mu-n,-\nu-n)}(x)\right|^{2} t^{n}=S_{+}^{-\mu} S_{-}^{-\nu} F\left(\nu, \mu ; \mu+\nu ;-\frac{t}{S_{+} S_{-}}\right),
$$

for $\mu+\nu>0$. Here, we have defined

$$
S_{ \pm}=1-|x \pm 1|^{2} t / 4=1-\left|\tau_{\mp}\right|^{2} .
$$

Therefore, we obtain the closed expression for the normalization factor:

$$
\mathcal{N}=S_{+}^{-k+r} S_{-}^{-k-r} F\left(k+r, k-r ; 2 k ;-\frac{t}{S_{+} S_{-}}\right)
$$

If $\left|\tau_{-}\right| \geq 1$ or $\left|\tau_{+}\right| \geq 1$ [i.e., $r=k+l$ or $r=-(k+l)$, respectively], we can use the relation between the hypergeometric function and the Jacobi polynomials (Erdélyi et al., 1953, Vol. 2, Sec. 10.8). For $l$ being a nonnegative integer and $\alpha>-1$, this relation can be written in the form

$$
F(-l, l+\alpha+\beta+1 ; \alpha+1 ; z)=\frac{l ! \Gamma(\alpha+1)}{\Gamma(l+\alpha+1)} P_{l}^{(\alpha, \beta)}(1-2 z) .
$$

Then we obtain

$$
\mathcal{N}=\frac{l ! \Gamma(2 k)}{\Gamma(2 k+l)} S_{i}^{l} S_{i^{\prime}}^{-2 k-l} P_{l}^{(2 k-1,0)}\left(1+\frac{2 t}{S_{+} S_{-}}\right)
$$

where $\left(i, i^{\prime}\right)=(+,-)$ for $r=k+l$ and $\left(i, i^{\prime}\right)=(-,+)$ for $r=-(k+l)$. It can be easily verified that for $r= \pm k$ (i.e., $l=0$ ), these formulas reduce to the corresponding results for the standard coherent state $\left|k, \zeta_{0}\right\rangle$ with $\zeta_{0}=-\tau_{ \pm}$, respectively.

Analogously to the $\mathrm{SU}(2)$ case, the above analytic expressions can be used for calculations of quantum statistical properties of the $\mathrm{SU}(1,1)$ AES. Here, we derive analytic expressions for moments of the generator $K_{3}$. By using the property $K_{3}|k, n\rangle=(k+n)|k, n\rangle$ and formula (4.29), moments of $K_{3}$ over the AES can be expressed as derivatives of $\mathcal{N}$ with respect to $t$ :

$$
\begin{gathered}
\left\langle K_{3}\right\rangle=\frac{t}{\mathcal{N}} \frac{\partial \mathcal{N}}{\partial t}+k \\
\left(\Delta K_{3}\right)^{2}=\frac{t^{2}}{\mathcal{N}} \frac{\partial^{2} \mathcal{N}}{\partial t^{2}}+\frac{t}{\mathcal{N}} \frac{\partial \mathcal{N}}{\partial t}-\left(\frac{t}{\mathcal{N}} \frac{\partial \mathcal{N}}{\partial t}\right)^{2}
\end{gathered}
$$


By using the formula (Erdélyi et al., 1953, Vol. 1, Sec. 2.1.2)

$$
\frac{d F(a, b ; c ; z)}{d z}=\frac{a b}{c} F(a+1, b+1 ; c+1 ; z)
$$

and the hypergeometric equation, we obtain exact analytic expressions for the moments of $K_{3}$ :

$$
\begin{aligned}
\left\langle K_{3}\right\rangle=\frac{-k Y+r\left(S_{+}-S_{-}\right)}{S_{+} S_{-}}+\frac{\left(k^{2}-r^{2}\right) Y t}{2 k S_{+}^{2} S_{-}^{2}} \Theta \\
\left(\Delta K_{3}\right)^{2}=(k+r) \frac{1-S_{-}}{S_{-}^{2}}+(k-r) \frac{1-S_{+}}{S_{+}^{2}}-\frac{\left(k^{2}-r^{2}\right) Y^{2} t}{\left(S_{+} S_{-}+t\right) S_{+}^{2} S_{-}^{2}} \\
-\frac{\left(k^{2}-r^{2}\right) t}{2 k S_{+}^{3} S_{-}^{3}}\left(\frac{S_{+} S_{-} Y^{2}}{S_{+} S_{-}+t}-2 k Y^{2}+Z\right) \Theta-\frac{\left(k^{2}-r^{2}\right)^{2} Y^{2} t^{2}}{4 k^{2} S_{+}^{4} S_{-}^{4}} \Theta^{2} .
\end{aligned}
$$

Here, $Y$ and $Z$ are given by equations (3.42) and (3.43), respectively [but with $S_{ \pm}$of equation (4.31)], and $\Theta$ is defined as

$$
\Theta=\left[F\left(k+r, k-r ; 2 k ;-\frac{t}{S_{+} S_{-}}\right)\right]^{-1} F\left(k+r+1, k-r+1 ; 2 k+1 ;-\frac{t}{S_{+} S_{-}}\right) .
$$

Note that the transition between the $\mathrm{SU}(2)$ and $\mathrm{SU}(1,1)$ cases can be formally performed by the interchange:

$$
j \leftrightarrow-k, \quad m_{0} \leftrightarrow r, \quad t \leftrightarrow-t, \quad\left(j+\left|m_{0}\right|\right) \Omega \leftrightarrow \frac{k^{2}-r^{2}}{2 k} \Theta .
$$

For $r= \pm(k+l)$ with $l>0$, by using relation (4.33), we find

$$
\Theta=\frac{2 k}{l}\left[P_{l}^{(2 k-1,0)}\left(1+\frac{2 t}{S_{+} S_{-}}\right)\right]^{-1} P_{l-1}^{(2 k, 1)}\left(1+\frac{2 t}{S_{+} S_{-}}\right) .
$$

For $r= \pm k$ (i.e., $l=0$ ), we obtain $\Theta=0$, and then we recover the known results for the SU(1,1) CS (Wodkiewicz and Eberly, 1985). The expressions for $\left\langle K_{3}\right\rangle$ and $\left(\Delta K_{3}\right)^{2}$ are significantly simplified in the case $Y=0$. This condition is satisfied if equation (3.45) holds, e.g., for $\beta_{1}=a \beta_{2}$ where $a$ is any real number (including the case when $\beta_{1}$ or $\beta_{2}$ vanishes). Then we obtain

$$
\begin{gathered}
\left\langle K_{3}\right\rangle=\frac{h+1}{h-1} r, \\
\left(\Delta J_{3}\right)^{2}=\frac{2 k h}{(h-1)^{2}}+\frac{\left(k^{2}-r^{2}\right) h^{2} t}{k(h-1)^{4}} \Theta,
\end{gathered}
$$

where $h$ is defined by equation (3.48). Note that the curve $\left|\tau_{-}\right|=1 /\left|\tau_{+}\right|$(that is, $Y=0$ ) lies in the allowed region of the parameter space (more specifically, in the discrete-spectrum region), except for the forbidden point $\left|\tau_{-}\right|=\left|\tau_{+}\right|=1$.

\subsection{Some special cases}

For $\beta_{+}=0$ and $\beta_{3} \neq 0$, we obtain $\tau_{-} \rightarrow \infty$, so we cannot use formula (4.18). In this case the solution of equation (4.16) is

$$
\Lambda(k, \lambda, \vec{\beta} ; \zeta)=\mathcal{N}^{-1 / 2} \zeta^{l}\left(1+\tau_{+} \zeta\right)^{-2 k-l},
$$

where $\tau_{+}=\beta_{1} / \beta_{3}$ and $l=-k+\lambda / \beta_{3}$. The condition of the analyticity requires $l=0,1,2, \ldots$ [i.e., the spectrum $\lambda=(k+l) \beta_{3}$ is discrete] and $\left|\tau_{+}\right|<1$. The decomposition over the orthonormal 
basis and quantum statistical properties of the AES can be obtained in this case by expanding the function (4.45) into the power series. We find

$$
\begin{gathered}
|k, \lambda, \vec{\beta}\rangle=\mathcal{N}^{-1 / 2} \sum_{n=l}^{\infty}\left[\frac{n ! \Gamma(2 k+n)}{l ! \Gamma(2 k+l)}\right]^{1 / 2} \frac{\left(-\tau_{+}\right)^{n-l}}{(n-l) !}|k, n\rangle, \\
\mathcal{N}=F\left(l+1, l+2 k ; 1 ;\left|\tau_{+}\right|^{2}\right)=\left(1-\left|\tau_{+}\right|^{2}\right)^{-2 k-l} P_{l}^{(0,2 k-1)}\left(\frac{1+\left|\tau_{+}\right|^{2}}{1-\left|\tau_{+}\right|^{2}}\right) .
\end{gathered}
$$

For $l=0$, the function (4.45) represents the standard coherent state $\left|k, \zeta_{0}\right\rangle$ with $\zeta_{0}=-\tau_{+}$. The corresponding eigenvalue equation is

$$
\left(K_{3}-\zeta_{0} K_{+}\right)\left|k, \zeta_{0}\right\rangle=k\left|k, \zeta_{0}\right\rangle
$$

For $\beta_{-}=0$ and $\beta_{3} \neq 0$, we can use the general results of sections 4.2 and 4.3 , with $b=\beta_{3}$, $\tau_{+}=0$ and $\tau_{-}=\beta_{3} / \beta_{1}$. This gives $\kappa=-\tau_{-}=-\beta_{3} / \beta_{1}, x=1, S_{+}=1-t\left(\right.$ where $\left.t=|\kappa|^{2}\right)$ and $S_{-}=1$. The corresponding analytic function is

$$
\Lambda(k, \lambda, \vec{\beta} ; \zeta)=\mathcal{N}^{-1 / 2}\left(1+\tau_{-} \zeta\right)^{-k+r},
$$

where $r=\lambda / \beta_{3}$. For $\left|\tau_{-}\right|<1$, this function is always analytic and $\lambda$ can take any complex value. In the special case $r=-k$ (i.e., $\lambda=-\beta_{3} k$ ), the function (4.49) represents the standard coherent state $\left|k, \zeta_{0}\right\rangle$ with $\zeta_{0}=-\tau_{-}$. The corresponding eigenvalue equation is

$$
\left(K_{3}-\zeta_{0}^{-1} K_{-}\right)\left|k, \zeta_{0}\right\rangle=-k\left|k, \zeta_{0}\right\rangle .
$$

For $\left|\tau_{-}\right| \geq 1$, the analyticity condition requires $r=k+l$ [i.e., the spectrum $\lambda=(k+l) \beta_{3}$ is discrete]. For $r=k(l=0)$, we have $\Lambda(\zeta)=1$ that represents the state $|k, 0\rangle$.

We next consider the degenerate case $b=0$. If $\beta_{+}$vanishes, then $\beta_{3}$ vanishes too, and the corresponding algebra element is just $K_{+}$. It can be easily verified that this operator has not any eigenstate. If $\beta_{-}$vanishes, then $\beta_{3}$ vanishes too, and the corresponding algebra element is just $K_{-}$. Its eigenstates are represented by the analytic function

$$
\Lambda(k, \lambda, \vec{\beta} ; \zeta)=\mathcal{N}^{-1 / 2} \exp (\lambda \zeta) .
$$

The eigenvalue $\lambda$ can take any complex value. By using equation 4.11, we find the normalization factor

$$
\mathcal{N}=\Gamma(2 k) I_{2 k-1}(2|\lambda|) \lambda^{1-2 k},
$$

where $I_{\nu}(x)$ is the $\nu$-order modified Bessel function of the first kind. The eigenstates of the lowering operator $K_{-}$were first constructed by Barut and Girardello (1971). The analytic representation based on these states will be discussed in section 4.6.

If $b=0$ and $\beta_{3} \neq 0$, the solution of equation (4.16) is

$$
\Lambda(k, \lambda, \vec{\beta} ; \zeta)=\mathcal{N}^{-1 / 2}(1+\tau \zeta)^{-2 k} \exp \left(-\frac{2 \lambda}{\beta_{3}} \frac{1}{1+\tau \zeta}\right)
$$

where

$$
\tau=\frac{2 \beta_{-}}{\beta_{3}}=\frac{\beta_{3}}{2 \beta_{+}} .
$$

The condition of the analyticity requires $|\tau|<1$. If this condition is satisfied, $\lambda$ can take any complex value. The decomposition of the corresponding AES over the orthonormal basis is obtained in section 4.6 by using the Barut-Girardello analytic representation [see equations (4.81) and (4.82) ]. Analogously to the general case, there are operators whose eigenstates are unnormalizable in sense of equation (4.11), but these states can be orthonormalized by the delta function. 
An example is the operator $K_{1}+K_{3}$, for which $|\tau|=1$ (Lindblad and Nagel, 1970; Nagel, 1995). In a special case $\lambda=0$, the analytic function is

$$
\Lambda(k, \lambda=0, \vec{\beta} ; \zeta)=\mathcal{N}^{-1 / 2}(1+\tau \zeta)^{-2 k}
$$

This function represents the standard coherent state $\left|k, \zeta_{0}\right\rangle$ with $\zeta_{0}=-\tau$ and $\mathcal{N}=\left(1-\left|\zeta_{0}\right|^{2}\right)^{-2 k}$.

For example, we can choose $\vec{\beta}=\left(1+\zeta_{0}^{2},-i\left(1-\zeta_{0}^{2}\right),-2 \zeta_{0}\right)$. Then the standard CS satisfy the eigenvalue equation

$$
\left(K_{-}-2 \zeta_{0} K_{3}+\zeta_{0}^{2} K_{+}\right)\left|k, \zeta_{0}\right\rangle=0
$$

\subsection{The $\mathrm{SU}(1,1)$ intelligent states}

The SU(1,1) generalized IS were defined by Trifonov (1994) as the eigenstates of the operator $\eta K_{1}-i K_{2}$ [see equation (2.15)]. In our notation, the generalized IS are the AES with $\vec{\beta}=$ $(\eta,-i, 0)$. For $\eta^{2} \neq 1$, the corresponding analytic function is given by the particular case of equation (4.18) with $b=\sqrt{1-\eta^{2}}$ and

$$
\tau_{ \pm}=\mp \sqrt{\frac{1-\eta}{1+\eta}} .
$$

The condition of the analyticity requires

$$
\left|\frac{1-\eta}{1+\eta}\right|<1 \Leftrightarrow \operatorname{Re} \eta>0
$$

In the allowed region of the parameter space $(\operatorname{Re} \eta>0)$, all the results of section 4.3 are valid, with $\kappa=2 \tau_{+}, x=0$, and $S_{+}=S_{-}=1-\left|\tau_{+}\right|^{2}$. For $\eta=0$, the algebra element is $K_{2}$ whose eigenstates do not possess a finite norm. The generalized intelligent state is also the standard coherent state when $r= \pm k$. The corresponding coherent-state amplitude is $\zeta_{0}=-\tau_{ \pm}$, respectively. Since $\eta$ is a complex number, $\zeta_{0}$ can acquire any complex value in the unit disk (excluding zero because $\eta \neq 1$ ). For $\eta=1$, the generalized IS are reduced to the BG states (the eigenstates of $K_{-}$). When the eigenvalue vanishes, $\lambda=0$, the BG states degenerate to the state $|k, 0\rangle$ that is the standard coherent state with $\zeta_{0}=0$. Therefore, the standard set of the $\mathrm{SU}(1,1) \mathrm{CS}$ is a subset of the $\mathrm{SU}(1,1)$ generalized IS.

We also briefly discuss three types of the SU(1,1) ordinary IS. The $K_{1}-K_{2}$ IS are determined, according to equation (2.16), as the eigenstates of the operator $K_{1}+i \gamma K_{2}$, where $\gamma$ is a real parameter. These states are the $\mathrm{SU}(1,1)$ AES with $\vec{\beta}=(1, i \gamma, 0)$. For $\gamma=1$, the solution does not exist since the raising operator $K_{+}$has not any eigenstate. For $\gamma=-1$, we obtain the BG states. For $\gamma^{2} \neq 1$, the corresponding analytic function is given by the particular case of equation (4.18) with $b=\sqrt{\gamma^{2}-1}$ and

$$
\tau_{ \pm}= \pm \sqrt{\frac{\gamma+1}{\gamma-1}}
$$

The condition of the analyticity requires

$$
\left|\frac{\gamma+1}{\gamma-1}\right|<1 \Leftrightarrow \gamma<0 .
$$

All the results of section 4.3 are valid here with $\kappa=2 \tau_{+}$and $x=0$. We also find that $S_{+}=S_{-}=$ $2|\gamma| /(1+|\gamma|)$ for $|\gamma|<1$, and $S_{+}=S_{-}=2 /(1+|\gamma|)$ for $|\gamma|>1$. For $\gamma=0$, the algebra element is $K_{1}$ whose eigenstates do not possess a finite norm. The $K_{1}-K_{2}$ IS coincide with the standard $\mathrm{CS}$ for $r= \pm k$. The corresponding coherent-state amplitude is $\zeta_{0}=-\tau_{ \pm}$, respectively. Since $\gamma$ is real, $\zeta_{0}$ is real for $|\gamma|>1$ and pure imaginary for $|\gamma|<1$. Therefore the set of the $K_{1}-K_{2}$ IS and the standard set of the $\mathrm{SU}(1,1)$ CS have an intersection. 
The $K_{1}-K_{3}$ IS are determined, according to equation (2.16), as the eigenstates of the operator $K_{1}+i \gamma K_{3}$. These states are the SU(1,1) AES with $\vec{\beta}=(1,0, i \gamma)$. The corresponding analytic function is given by the particular case of equation (4.18) with $b=i \sqrt{\gamma^{2}+1}$ and

$$
\tau_{ \pm}=-i\left(\gamma \pm \sqrt{\gamma^{2}+1}\right)^{-1}
$$

The analyticity condition requires $r=k+l$ for $\left|\tau_{-}\right|>1$ (i.e., for $\gamma>0$ ), and $r=-(k+l)$ for $\left|\tau_{+}\right|>1$ (i.e., for $\gamma<0$ ). Here, as usual, $l=0,1,2, \ldots$ This condition can be expressed in the form

$$
\lambda=i(\operatorname{sgn} \gamma)(k+l) \sqrt{\gamma^{2}+1},
$$

(For $\gamma=0$, we have $\left|\tau_{+}\right|=\left|\tau_{-}\right|=1$, and the algebra element is $K_{1}$ with unnormalizable eigenstates.) All the results of section 4.3 are valid here with $\kappa=-2 i \sqrt{\gamma^{2}+1}$ and $x=\gamma / \sqrt{\gamma^{2}+1}$. Note that $\left|\tau_{+} \tau_{-}\right|=1$, and therefore we can use simple expressions (4.43) and (4.44), with $h=$ $2 \gamma^{2}+2 \sqrt{\gamma^{2}+1}+1$. The intersection between the $K_{1}-K_{3}$ IS and the standard CS is obtained for $l=0$, i.e., $\lambda= \pm i k \sqrt{\gamma^{2}+1}$. The corresponding coherent-state amplitude is $\zeta_{0}=-\tau_{ \pm}$, respectively. Since $\gamma$ is real, $\zeta_{0}$ is pure imaginary.

The $K_{2}-K_{3}$ IS are determined, according to equation (2.16), as the eigenstates of the operator $K_{2}+i \gamma K_{3}$. These states are the SU(1,1) AES with $\vec{\beta}=(0,1, i \gamma)$. The corresponding analytic function is given by the particular case of equation (4.18) with $b=i \sqrt{\gamma^{2}+1}$ and

$$
\tau_{ \pm}=-\left(\gamma \pm \sqrt{\gamma^{2}+1}\right)^{-1}
$$

The analyticity condition here is the same as in the preceding case [see equation (4.62)]. All the results of section 4.3 are valid here with $\kappa=-2 \sqrt{\gamma^{2}+1}$ and $x=\gamma / \sqrt{\gamma^{2}+1}$. Once again, $\left|\tau_{+} \tau_{-}\right|=1$, and therefore we can use simple expressions (4.43) and (4.44), with $h=2 \gamma^{2}+$ $2 \sqrt{\gamma^{2}+1}+1$. The intersection between the $K_{2}-K_{3}$ IS and the standard CS is obtained for $l=0$, i.e., $\lambda= \pm i k \sqrt{\gamma^{2}+1}$. The corresponding coherent-state amplitude is $\zeta_{0}=-\tau_{ \pm}$, respectively. Since $\gamma$ is real, $\zeta_{0}$ is also real.

\subsection{The Barut-Girardello analytic representation}

The BG states (Barut and Girardello, 1971) are defined as the eigenstates of the lowering operator $K_{-}$:

$$
K_{-}|k, z\rangle=z|k, z\rangle,
$$

where $z$ is an arbitrary complex number. The expansion of these states over the orthonormal basis is

$$
|k, z\rangle=\frac{z^{k-1 / 2}}{\sqrt{I_{2 k-1}(2|z|)}} \sum_{n=0}^{\infty} \frac{z^{n}}{\sqrt{n ! \Gamma(2 k+n)}}|k, n\rangle,
$$

where the eigenvalue $z$ is an arbitrary complex number. The BG states are normalized but they are not orthogonal to each other:

$$
\left\langle k, z_{1} \mid k, z_{2}\right\rangle=\frac{I_{2 k-1}\left(2 \sqrt{z_{1}^{*} z_{2}}\right)}{\sqrt{I_{2 k-1}\left(2\left|z_{1}\right|\right) I_{2 k-1}\left(2\left|z_{2}\right|\right)}} .
$$

The identity resolution is

$$
\int d \mu(k, z)|k, z\rangle\langle k, z|=I, \quad d \mu(k, z)=\frac{2}{\pi} K_{2 k-1}(2|z|) I_{2 k-1}(2|z|) d^{2} z,
$$


where $K_{\nu}(x)$ is the $\nu$-order modified Bessel function of the second kind. Thus the BG states form an overcomplete set. Therefore, for any state $|\Psi\rangle=\sum_{n=0}^{\infty} c_{n}|k, n\rangle$ in the Hilbert space, one can construct the analytic function

$$
f(z)=\frac{\sqrt{I_{2 k-1}(2|z|)}}{z^{k-1 / 2}}\left\langle k, z^{*} \mid \Psi\right\rangle=\sum_{n=0}^{\infty} \frac{c_{n}}{\sqrt{n ! \Gamma(2 k+n)}} z^{n} .
$$

Then the state $|\Psi\rangle$ can be represented in the BG basis

$$
\begin{gathered}
|\Psi\rangle=\int d \mu(k, z) \frac{\left(z^{*}\right)^{k-1 / 2}}{\sqrt{I_{2 k-1}(2|z|)}} f\left(z^{*}\right)|k, z\rangle, \\
\langle\Psi \mid \Psi\rangle=\int d \mu(k, z) \frac{|z|^{2 k-1}}{I_{2 k-1}(2|z|)}\left|f\left(z^{*}\right)\right|^{2}<\infty .
\end{gathered}
$$

The standard coherent state $|k, \zeta\rangle$ is represented by the function

$$
\mathcal{F}(k, \zeta ; z)=\frac{\sqrt{I_{2 k-1}(2|z|)}}{z^{k-1 / 2}}\left\langle k, z^{*} \mid k, \zeta\right\rangle=\frac{\left(1-|\zeta|^{2}\right)^{k}}{\sqrt{\Gamma(2 k)}} \exp (\zeta z)
$$

As has been recently shown (Brif et al., 1996), the BG representation and the analytic representation in the unit disk are related through a Laplace transform.

The operators $K_{ \pm}$and $K_{3}$ act in the Hilbert space of analytic functions $f(z)$ as linear differential operators

$$
K_{+}=z, \quad K_{-}=2 k \frac{d}{d z}+z \frac{d^{2}}{d z^{2}}, \quad K_{3}=z \frac{d}{d z}+k .
$$

By introducing the analytic function

$$
\Lambda(k, \lambda, \vec{\beta} ; z)=\frac{\sqrt{I_{2 k-1}(2|z|)}}{z^{k-1 / 2}}\left\langle k, z^{*} \mid k, \lambda, \vec{\beta}\right\rangle
$$

the eigenvalue equation (4.14) for the $\mathrm{SU}(1,1)$ AES $|k, \lambda, \vec{\beta}\rangle$ can be converted into the second-order linear homogeneous differential equation

$$
\left[\beta_{+} z\right] \frac{d^{2}}{d z^{2}} \Lambda(k, \lambda, \vec{\beta} ; z)+\left[\beta_{3} z+2 k \beta_{+}\right] \frac{d}{d z} \Lambda(k, \lambda, \vec{\beta} ; z)+\left[\beta_{-} z+k \beta_{3}-\lambda\right] \Lambda(k, \lambda, \vec{\beta} ; z)=0 .
$$

This equation can be transformed into the Kummer equation for the confluent hypergeometric function $\Phi(a ; c ; x)$ or into the Bessel equation, depending on the values of the parameters. Using the general results of Erdélyi et al. (1953, Vol. 1, Sec. 6.2), we find the solution of equation (4.74).

We first consider the general case $b \neq 0$. For $\beta_{+} \neq 0$, we have two independent solutions:

$$
\begin{aligned}
& \Lambda(k, \lambda, \vec{\beta} ; z)=\exp \left(-\tau_{ \pm} z\right) \Phi\left(k \mp r ; 2 k ; \mp b z / \beta_{+}\right), \\
& \tilde{\Lambda}(k, \lambda, \vec{\beta} ; z)=\exp \left(-\tau_{ \pm} z\right) z^{1-2 k} \Phi\left(\tilde{k} \mp r ; 2 \tilde{k} ; \mp b z / \beta_{+}\right)
\end{aligned}
$$

where $\tau_{ \pm}$is defined by equation (4.19), $r=\lambda / b$, and $\tilde{k}=1-k$. The first solution $\Lambda$ is always analytic, but the second solution $\tilde{\Lambda}$ is not (except for $k=\frac{1}{2}$ when the two solutions coincide). [Another special case is $k=\frac{1}{4}$ and $\tilde{k}=\frac{3}{4}$ (or vice versa). These representations do not belong to the discrete series, but they occur for the so-called two-photon realization of the su(1,1) Lie algebra. This realization is important in quantum optics, as it provides the mathematical model of squeezing of the single-mode quantized light field. The corresponding analytic representations have been recently studied in detail (Brif et al., 1996; Brif, 1996).] Here, we consider only the discrete-series representations ( $k$ is a positive integer or half-integer) and hence use only the first 
solution $\Lambda(z)$. Also, the solution should be normalizable, i.e., the integral in equation (4.70) must be convergent. The convergence of this integral can be easily analyzed by using the asymptotic expansion of the integrand. It is remarkable that the normalization condition for the function $\Lambda(z)$ of equation (4.75) is equivalent to the analyticity condition for the function $\Lambda(\zeta)$ of equation (4.18) [see the discussion after equation (4.20) and Fig. 1]. Actually, these functions are related through a Laplace transform (Brif et al., 1996). The upper and lower signs in equation (4.75) are equivalent, because the confluent hypergeometric function can be written in two equivalent forms which are related by Kummer's transformation (Erdélyi et al., 1953, Vol. 1, Sec. 6.3):

$$
\Phi(a ; c ; x)=e^{x} \Phi(c-a ; c ;-x) .
$$

Kummer's transformation in equation (4.75) is equivalent to the replacement $b \leftrightarrow-b$. For $\lambda= \pm k b$, the AES coincide with the standard CS. Then equation (4.75) reads

$$
\Lambda(k, \lambda, \vec{\beta} ; z)=\mathcal{N}^{-1 / 2} \exp \left(-\tau_{ \pm} z\right) .
$$

The corresponding coherent-state amplitude is $\zeta_{0}=-\tau_{ \pm}$.

In the case $\beta_{+}=0$ and $\beta_{3} \neq 0$, the solution of equation (4.74) is

$$
\Lambda(k, \lambda, \vec{\beta} ; z)=\mathcal{N}^{-1 / 2} z^{l} \exp \left(-\tau_{+} z\right),
$$

where $\tau_{+}=\beta_{1} / \beta_{3}$ and $l=-k+\lambda / \beta_{3}$. The condition of the analyticity requires $l=0,1,2, \ldots$, and the normalization condition is $\left|\tau_{+}\right|<1$. This is in full agreement with the analyticity condition for the function $\Lambda(\zeta)$ of equation (4.45).

We next consider the degenerate case $b=0$. If $\beta_{+}$vanishes, equation (4.74) has not any nontrivial analytic solution, and consequently the operator $K_{+}$has not any eigenstate. For $\beta_{+} \neq 0$, the analytic solution of equation (4.74) is

$$
\Lambda(k, \lambda, \vec{\beta} ; z)=\mathcal{N}^{-1 / 2}\left(\lambda^{\prime} z\right)^{1 / 2-k} I_{2 k-1}\left(2 \sqrt{\lambda^{\prime} z}\right) \exp (-\tau z),
$$

where $\tau$ is defined by equation (4.54) and $\lambda^{\prime}=\lambda / \beta_{+}$. The normalization condition requires $|\tau|<1$, in accordance with the analyticity condition for the function $\Lambda(\zeta)$ of equation (4.53). The decomposition of the AES with $b=0$ over the orthonormal basis and the corresponding normalization factor can be obtained by expanding the analytic function $\Lambda(z)$ of equation (4.80) into the power series. By using generating functions for the Laguerre polynomials $L_{n}^{\alpha}(x)$ (Erdélyi et al., 1953, Vol. 2, Sec. 10.12; Srivastava and Manocha, 1984, Sec. 2.5), we find

$$
\begin{gathered}
|k, \lambda, \vec{\beta}\rangle=\mathcal{N}^{-1 / 2} \sum_{n=0}^{\infty}\left[\frac{n !}{\Gamma(2 k+n)}\right]^{1 / 2} L_{n}^{2 k-1}\left(\lambda^{\prime} / \tau\right)(-\tau)^{n}|k, n\rangle, \\
\mathcal{N}=\frac{\left|\lambda^{\prime}\right|^{1-2 k}}{1-|\tau|^{2}} I_{2 k-1}\left(\frac{2\left|\lambda^{\prime}\right|}{1-|\tau|^{2}}\right) \exp \left[-\frac{2|\tau|^{2} \operatorname{Re}\left(\lambda^{\prime} / \tau\right)}{1-|\tau|^{2}}\right] .
\end{gathered}
$$

In a special case $\lambda=0$, the corresponding AES are the standard CS $\left|k, \zeta_{0}\right\rangle$ with $\zeta_{0}=-\tau$. If $\beta_{-}$vanishes, $\beta_{3}$ vanishes too, and the corresponding algebra element is just $K_{-}$. Then equation (4.80) reads

$$
\Lambda(k, \lambda, \vec{\beta} ; z)=\mathcal{N}^{-1 / 2} \frac{I_{2 k-1}(2 \sqrt{\lambda z})}{(\lambda z)^{k-1 / 2}} .
$$

This function represents the BG state $\left|k, z_{0}\right\rangle$ with $z_{0}=\lambda$, and the normalization factor is

$$
\mathcal{N}=z_{0}^{1-2 k} I_{2 k-1}\left(2\left|z_{0}\right|\right) \text {. }
$$

One can also use the general solution (4.75) in order to consider the particular case of the $\mathrm{SU}(1,1)$ IS. The BG representation of the $\mathrm{SU}(1,1)$ generalized IS was first obtained by Trifonov (1994). His results can be reproduced by taking $\vec{\beta}=(\eta,-i, 0)$ that corresponds to the algebra element $\eta K_{1}-i K_{2}$. It is not difficult to obtain also the BG analytic representation for different types of the $\mathrm{SU}(1,1)$ ordinary IS. 


\section{CONCLUSIONS}

In this paper we have shown that the algebra-eigenstate formalism unifies the descriptions of various kinds of states within a common frame. This clarifies relations between different types of states and the physical basis of their mathematical properties. The use of an analytic representation enables us to write a linear homogeneous differential equation that determines all the kinds of the AES. Often, this is a first-order equation that can be immediately integrated, and then we derive analytic functions representing the AES. These functions yield all the information about the AES. For example, we have presented here a method for the calculation of exact analytic expressions for quantum statistical properties of the AES. This method should be useful in physical applications, especially in the field of quantum optics. On the other hand, the analytic representations provide us with a simple and effective way to find different subsets of the AES and to analyse the relations between them. We have determined, for instance, the conditions to obtain the standard CS and the generalized and ordinary IS.

In the present work we have concentrated on the most elementary simple Lie groups, but the theory of the AES is in general applicable to arbitrary Lie groups describing a wide class of quantum systems. Therefore, the algebra-eigenstate formalism can find applications in many fields of modern quantum physics. We mention, for example, quantum optics where the AES can provide a general view on the problem of squeezing with practical applications to the reduction of quantum fluctuations and the improvement of the accuracy of interferometric measurements.

\section{ACKNOWLEDGEMENTS}

I wish to thank my colleagues A. Berengolts, K. Gokhberg, A. Kenis and M. Lublinsky for their interest in my work and many stimulating discussions. The financial help from the Technion is gratefully acknowledged. 


\section{REFERENCES}

Agarwal, G. S. (1988). Journal of Optical Society of America B, 5, 1940.

Agarwal, G. S., and Puri R. R. (1990). Physical Review A, 41, 3782.

Aragone, C., Guerri, G., Salamo, S., and Tani, J. L. (1974). Journal of Physics A, 7, L149.

Aragone, C., Chalbaud, E., and Salamo, S. (1976). Journal of Mathematical Physics, 17, 1963.

Bargmann, V. (1947). Annals of Mathematics, 48, 568.

Bargmann, V. (1961). Communications in Pure and Applied Mathematics, 14, 187.

Barut, A. O., and Girardello, L. (1971). Communications in Mathematical Physics, 21, 41.

Berezin, F. A. (1975). Communications in Mathematical Physics, 40, 153.

Bergou, J. A., Hillery, M., and Yu, D. (1991). Physical Review A, 43, 515.

Brif, C. (1995). Quantum and Semiclassical Optics, 7, 803.

Brif, C. (1996). Annals of Physics, 251, 180.

Brif, C., and Ben-Aryeh, Y. (1994a). Journal of Physics A, 27, 8185.

Brif, C., and Ben-Aryeh, Y. (1994b). Quantum Optics, 6, 391.

Brif, C., and Ben-Aryeh, Y. (1996). Quantum and Semiclassical Optics, 8, 1.

Brif, C., and Mann, A. (1996a). Physics Letters A, 219, 257.

Brif, C., and Mann, A. (1996b). Physical Review A, 54, 4505.

Brif, C., Vourdas, A., and Mann, A. (1996). Journal of Physics A, 29, 5873.

Bužek, V. (1990). Journal of Modern Optics, 37, 303.

Dodonov, V. V., Malkin, I. A., and Man'ko, V. I. (1974). Physica, 72, 597.

Dodonov, V. V., Kurmyshev, E. V., and Man'ko, V. I. (1980). Physics Letters A, 79, 150.

Erdélyi, A. et al., eds. (1953). Higher Transcendental Functions: Bateman Manuscript Project, McGraw-Hill, New York.

Fock, V. A. (1928). Zeitschrift für Physik, 49, 339.

Gerry, C. C., and Grobe, R. (1995). Physical Review A, 51, 4123.

Gilmore, R. (1972). Annals of Physics, 74, 391.

Gilmore, R. (1974). Journal of Mathematical Physics, 15, 2090.

Glauber, R. J. (1963). Physical Review, 131, 2766.

Hillery, M. (1987). Physical Review A, 36, 3796.

Hillery, M. (1989). Physical Review A, 40, 3147.

Hillery, M, and Mlodinow, L. (1993). Physical Review A, 48, 1548. 
Jackiw, R. (1968). Journal of Mathematical Physics, 9, 339.

Klauder, J. R., and Skagerstam, B. S. (1985). Coherent states: Applications in Physics and Mathematical Physics, World Scientific, Singapore.

Lindblad, G., and Nagel, B. (1970). Annales de l'Institut Henri Poincaré A, 13, 27.

Luis, A., and Peřina, J. (1996). Physical Review A, 53, 1886.

Nagel, B. (1995). In Modern Group Theoretical Methods in Physics, J. Bertrand et al., eds., Kluwer, Dordrecht, pp. 211-220.

Nieto, M. M., and Truax, D. (1993). Physical Review Letters, 71, 2843.

Perelomov, A. M. (1972). Communications in Mathematical Physics, 26, 222.

Perelomov, A. M. (1977). Soviet Physics Uspekhi, 20, 703.

Perelomov, A. M. (1986). Generalized Coherent States and Their Applications, Springer, Berlin.

Prakash, G. S., and Agarwal, G. S. (1994). Physical Review A, 50, 4258.

Prakash, G. S., and Agarwal, G. S. (1995). Physical Review A, 52, 2335.

Puri, R. R. (1994). Physical Review A, 49, 2178.

Puri, R. R., and Agarwal, G. S. (1996). Physical Review A, 53, 1786.

Rasetti, M. (1975). International Journal of Theoretical Physics, 13, 425.

Ruschin, S., and Ben-Aryeh, Y. (1976). Physics Letters A, 58, 207.

Schrödinger, E. (1926). Die Naturwissenschaften, 14, 664.

Segal, I. E. (1962). Illinois Journal of Mathematics, 6, 500.

Solomon, A. I. (1971). Journal of Mathematical Physics, 12, 390.

Srivastava, H. M., and Manocha, H. L. (1984). A Treatise on Generating Functions, Ellis Horwood, Chichester.

Stoler, D. (1970). Physical Review D, 1, 3217.

Stoler, D. (1971). Physical Review D, 4, 2308.

Trifonov, D. A. (1994). Journal of Mathematical Physics, 35, 2297.

Trifonov, D. A. (1996a). Preprint, quant-ph/9609001.

Trifonov, D. A. (1996b). Preprint, quant-ph/9609017.

Vanden-Bergh, G., and DeMeyer, H. (1978). Journal of Physics A, 11, 1569.

Vilenkin, N. Ya. (1968). Special Functions and the Theory of Group Representations, American Mathematical Society, Providence, Rhode Island.

Weyl, H. (1950). The Theory of Groups and Quantum Mechanics, Dover, New York.

Wodkiewicz, K., and Eberly, J. H. (1985). Journal of Optical Society of America B, 2, 458.

Yu, D., and Hillery, M. (1994). Quantum Optics, 6, 37. 
Yuen, H. (1976). Physical Review A, 13, 2226.

Zhang, W. M., Feng, D. H., and Gilmore, R. (1990). Reviews of Modern Physics, 62, 867. 